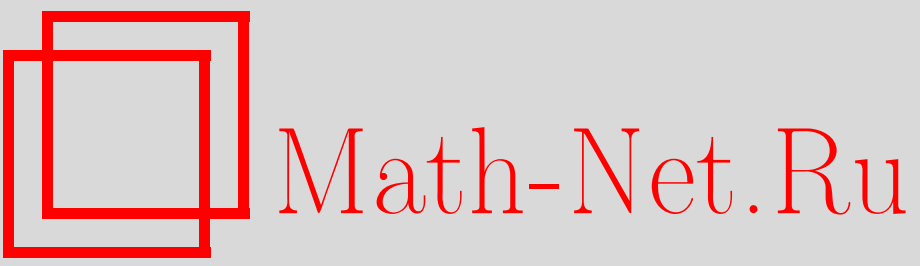

В. Р. Фаталов, О методе Лапласа для гауссовских мер в банаховом пространстве, Теория вероятн. и ее примен., 2013, том 58, выпуск 2, 325-354

DOI: https://doi.org/10.4213/tvp4509

Использование Общероссийского математического портала Math-Net.Ru подразумевает, что вы прочитали и согласны с пользовательским соглашением

http://www.mathnet.ru/rus/agreement

Параметры загрузки:

IP: 54.198 .55 .26

26 апреля 2023 г., $07: 49: 37$ 


\section{О МЕТОДЕ ЛАПЛАСА ДЛЯ ГАУССОВСКИХ МЕР В БАНАХОВОМ ПРОСТРАНСТВЕ ${ }^{1)}$}

В статье доказаны результаты о точных асимптотиках вероятностей $P_{A}(u D), u \rightarrow \infty$, где $P_{A}$ - гауссовская мера в бесконечномерном банаховом пространстве $B$ со средним нуль и невырожденным ковариационным оператором $A, D=\{x \in B: Q(x) \geqslant 0\}-$ борелевское множество в $B, Q$ - гладкая функция. Изучен случай, когда функционал действия достигает своего минимума на $D$ на одномерном многообразии. Методом исследования является метод Лапласа в банаховых пространствах для гауссовских мер. На основе общего результата, полученного в статье, найдена при $0<p \leqslant 6$ точная асимптотика больших уклонений распределений $L^{p}$-функционалов для центрированного броуновского моста, возникающего в качестве предела при рассмотрении статистики Ватсона. Явные константы даны для случаев $p=1$ и $p=2$.

Ключевые слова и фразы: метод Лапласа, большие уклонения, гауссовские процессы, принцип больших уклонений, функционал действия, центрированный броуновский мост, статистика Ватсона, гипергеометрическая функция.

1. Введение и формулировка основных результатов. Во многих разделах теории вероятностей, математической статистики, статистической физики, функционального анализа возникает необходимость вычисления точной асимптотики модельного интеграла типа Лапласа

$$
J_{u}(D)=\int_{D} f(x) \exp \left\{-u^{2} F(x)\right\} d P_{u}(x)
$$

при $u \rightarrow \infty$. Здесь $D$ - борелевское множество в том или ином бесконечномерном банаховом пространстве $B, f$ и $F$ - действительные функции, определенные на $B, P_{u}$ - семейство вероятностных мер в $B$, зависящих от параметра $u$.

\footnotetext{
* Московский государственный университет, им. М. В. Ломоносова, механико-математический факультет, лаборатория теории вероятностей; e-mail: vrfatalov@yandex.ru

1) Работа выполнена при поддержке РФФИ (гранты № 07-01-00077, 11-01-00050).
} 
Наиболее распространенными и важными являются следующие два класса задач, связанные с интегралом (1.1). Первый класс задач, представляющий большое значение для проблем статистической и математической физики, - это когда в качестве множества $D$ берется все пространство $B$. Оказалось, что для достаточно гладких функций $f, F$ и $Q$ точную асимптотику интегралов $J_{u}(B)$ можно находить с помощью асимптотического метода в функциональном пространстве, который аналогичен методу Лапласа в $\mathbf{R}^{k}$. Впервые на это обратил внимание Р. Фейнман в своих работах по квантовой физике [38].

Становление метода Лапласа в бесконечномерных пространствах как строгого математического метода произошло в восьмидесятые годы прошлого века, после появления и развития асимптотического принципа больших уклонений (ПБУ) М.Д. Донскера-С. Варадана в теории вероятностей (см. [4], [7, гл. 3], [20, §12], [26, гл. 2], [46], [60]). Именно, в работах Р. Эллиса, Дж. Розена [47] (гауссовские меры), Р. Азенкотта [39], Ж. Бен Аруса [40] (диффузионные процессы с малым параметром), Э. Больтхаузена [41] (распределения сумм независимых банаховозначных случайных элементов), С. Кусуоки, Й. Тамуры [51] (распределение времени пребывания марковского симметричного процесса) был обоснован в окончательной форме асимптотический метод Лапласа в банаховых пространствах, позволивший вычислять точные асимптотики $J_{u}(B)$ для дважды дифференцируемых по Фреше функций $F(x)$. Этой тематике посвящены также работы А.Д. Вентцеля, М. И. Фрейдлина [7], [8] (марковские процессы) и ряд других. Систематическому изложению этих результатов посвящена первая часть обзора [26].

Второй класс задач, связанный с интегралом (1.1) и имеющий большое значение для различных областей теории вероятностей, возникает при $f(x) \equiv 1$ и $F(x) \equiv 0$. В качестве множества $D$, как правило, фигурирует «хорошее» множество вида

$$
D=\{x: Q(x) \geqslant 0\}
$$

где $Q(x)$ - непрерывный функционал на $B$. При этом множество $D$ должно иметь непустую внутренность и быть «близким» к выпуклому (см. условие (B2) ниже). В качестве функционала $Q(x)$ можно брать такие функционалы, как $\sup _{t \in T} x(t)-1, \int_{0}^{1}|x(t)|^{p} d t-1$, а в качестве банаховых пространств - пространства $C(T), L^{p}[0,1]$ и т.д. (см. [26]). В этом случае задача о вычислении точной асимптотики интеграла (1.1) превращается в задачу о нахождении точной асимптотики больших уклонений для вероятностей $P_{u}(D)$ при $u \rightarrow \infty$.

Гауссовские меры являются одним из самых важных примеров распределений, для которых возможно вычисление точной асимптотики интеграла (1.1). В этом случае рассматриваемое семейство гауссовских 
мер $P_{u}$ часто строится по одной заданной гауссовской мере $P$ и имеет простой вид: $P_{u}(D) \equiv P(u D)$, где $u D=\{u x: x \in D\}, u>u_{0}$.

Поведение точных асимптотик гауссовских мер $P(u D)$ существенно зависит от гладкости функционала $Q$. Для дифференцируемых функционалов $Q$ точные асимптотики гауссовских мер $P(u D)$ в случае, когда так называемый функционал действия имеет конечное число точек минимума, были впервые найдены автором в результате дальнейшего развития метода Лапласа в работах [26], [29], [34]. В статье [26, §3.3] были также доказаны соответствующие теоремы и о точных асимптотиках гауссовских интегралов $J_{u}(D)$. При этом, наряду с использованием результатов из статьи [47], были осуществлены и оригинальные построения по переносу некоторых конечномерных результатов из $[37$, гл. 2] на бесконечномерные пространства. Некоторые примеры применения общих результатов из [29], [34] рассмотрены в статьях [35], [36].

В данной работе мы докажем результаты о точных асимптотиках гауссовских мер $P(u D)$ в случае, когда функционал действия имеет одномерное многообразие точек минимума.

Для непрерывных, но не дифференцируемых функционалов $Q$ типа супремума разработана специфическая модификация метода Лапласа так называемый метод двойных сумм [26, гл. 2], [25], [30], [31], который позволяет находить точные асимптотики вероятностей

$$
\begin{aligned}
& \mathbf{P}\left\{\sup _{t \in T}|X(t)|>u\right\}, \\
& \mathbf{P}\left\{\sup _{t, s \in[0,1], t \neq s} \frac{|X(t)-X(s)|}{|t-s|^{\gamma}}>u\right\}, \quad 0 \leqslant \gamma<1, \quad u \rightarrow \infty,
\end{aligned}
$$

для широкого класса гауссовских полей $X(t)$, заданных на компакте $T \subset$ $\mathbf{R}^{k}, k \geqslant 1$.

В статьях [32], [48] было осуществлено построение метода Лапласа для вычисления точных асимптотик вероятностей $\mathbf{P}\left\{S_{n} / b_{n} \in D\right\}, n \rightarrow$ $\infty$, где $S_{n}$ - сумма $n$ независимых одинаково распределенных случайных элементов со значениями в банаховом пространстве $B, D-$ множество вида (1.2) с гладкой функцией $Q$ и $\left\{b_{n}\right\}$ - последовательность положительных чисел такая, что $b_{n} \equiv n$ (случай больших уклонений) или $b_{n} / \sqrt{n} \rightarrow \infty, b_{n} / n \rightarrow 0$ (случай умеренных уклонений). Построение метода Лапласа для вероятностей, связанных с распределением времени пребывания марковского симметричного процесса, выполнено в работе [33].

Среди недавних работ, посвященных асимптотикам типа Лапласа для вероятностных мер и интегралов, отметим работы [20], [45], [54], [61] (гауссовские меры), [50], [52], [53] (распределения сумм независимых банаховозначных случайных элементов), [42] (марковские процессы), [58], 
[59] (стохастические дифференциальные уравнения в частных производных).

Перейдем к изложению результатов, полученных в данной статье.

Пусть $(\mathbf{B},\|\cdot\|)$ - действительное сепарабельное банахово пространство, $\left(\mathbf{B}^{*},\|\cdot\|_{*}\right)$ - сопряженное пространство, $\langle\cdot, \cdot\rangle-$ билинейная форма, устанавливающая двойственность между $\mathbf{B}$ и $\mathbf{B}^{*}$.

Пусть $P_{A}$ - гауссовская мера, определенная на борелевской $\sigma$ алгебре пространства $\mathbf{B}$, со средним нуль и ковариационным оператором $A: \mathbf{B}^{*} \rightarrow \mathbf{B}$ (см. [6, гл. 4, §2, п. 4], [10, гл. 1]). Напомним, что ковариационный оператор $A$ является: симметричным, т.е. $\langle A x, y\rangle=\langle A y, x\rangle$ для всех $x, y \in \mathbf{B}^{*}$, положительным, т.е. $\langle A y, y\rangle \geqslant 0$ для всех $y \in \mathbf{B}^{*}$, ядерным, т.е. для некоторой последовательности $\left\{x_{k}\right\}$ элементов $\mathbf{B}$ такой, что $\sum_{k=1}^{\infty}\left\|x_{k}\right\|^{2}<\infty$, верно представление $A x^{*}=\sum_{k=1}^{\infty}\left\langle x_{k}, x^{*}\right\rangle x_{k}$, $x^{*} \in \mathbf{B}^{*}$ (см. [6, гл. $3, \S 2$, п. 3$]$ ).

Будем обозначать первую и вторую производные по Фреше числовой функции $S: \mathbf{B} \rightarrow \mathbf{R}$ в точке $x$ через $S^{\prime}(x)$ и $S^{\prime \prime}(x)$ (см. определение 1 в $[2$, п. 2.2.1]), при этом мы предполагаем, что эти производные можно рассматривать соответственно как элемент пространства $\mathbf{B}^{*}$ и как линейный оператор из $\mathbf{B}$ в $\mathbf{B}^{*}$. Дадим следующее определение.

О п р е д е л е н и е 1 . Пусть $U$ - открытое множество в $\mathbf{B}$ и $0<$ $\alpha<1$. Обозначим через $\operatorname{Hol}_{\alpha}^{2}(U)$ множество дважды дифференцируемых по Фреше на $U$ числовых функций $S: \mathbf{B} \rightarrow \mathbf{R}$, для которых

$$
\left\|S^{\prime \prime}(x)-S^{\prime \prime}(y)\right\|=O\left(\|x-y\|^{\alpha}\right), \quad\|x-y\| \rightarrow 0, \quad x, y \in U
$$

(вторая производная удовлетворяет на $U$ условию непрерывности по Гёльдеру порядка $\alpha$ ).

Здесь операторная норма также обозначена символом $\|\cdot\|$. Пусть $\operatorname{Dom}(C)$ обозначает область определения оператора $C$. Придерживаясь работ $[26, \S 3.3],[32],[47$, ч. II], введем в рассмотрение следующие условия.

(B1) Ковариачионный оператор $A$ инъективен, т.е. $\langle A y, y\rangle \equiv$ $\int_{\mathbf{B}}\langle x, y\rangle^{2} P_{A}(d x)>0$ для всех $0 \neq y \in \mathbf{B}^{*}$.

Отметим, что, согласно следствию из предложения 1.3 в [26, гл. 3 , $\S 1]$, ковариационный оператор $A$ инъективен тогда и только тогда, когда множество его значений $R(A):=\left\{A y: y \in \mathbf{B}^{*}\right\}$ всюду плотно в $(\mathbf{B},\|\cdot\|)$.

(B2) Множество $D$ замкнуто и имеет вид

$$
D=\{x \in \mathbf{B}: Q(x) \geqslant 0\},
$$

где $Q(x)$ - непрерьвная действительная функция такая, что одно из множеств $D, \mathbf{B} \backslash D$ представимо в виде конечного объединения непересекающихся выпукльх множеств из В. Пусть $D$ имеет непустую внутренность и не содержит нулевого элемента в $\mathbf{B}$. 
(B3) Квадратичная форма $\left\langle x, A^{-1} x\right\rangle$ достигает своего минимума $\sigma^{-2}>0$ на $D \cap R(A)$ на одномерном дважды дифферениируемом компактном многообразии $M$, лежащем на границе $\partial D$ множества $D$.

Таким образом,

$$
\left\langle m, A^{-1} m\right\rangle=: \sigma^{-2}>0 \quad \text { для всех } m \in M .
$$

Ниже тождественные операторы в различных пространствах обозначаются одним и тем же символом $\mathscr{I}$.

Обозначим через $n_{x} \in \mathbf{B}$ единичный касательный вектор к кривой $M$ в точке $x$, через $\widehat{M}_{x}$ - одномерное аффинное пространство, натянутое на вектор $n_{x}$ и содержащее точку $x$ (т.е. одномерное касательное пространство к $M$ в точке $x$ ), см. [2, гл. 2, п. 2.3.5], [22, гл. 3, §3]. Тогда $M_{x}:=\widehat{M}_{x}-x$ представляет собой одномерное подпространство в $\mathbf{B}$, содержащее точку 0 . Как это принято, мы будем рассматривать единичные касательные векторы как свободные векторы, поэтому выходящий из точки 0 единичный вектор, на который натянуто подпространство $M_{x}$, мы также будем обозначать через $n_{x}$.

Согласно общей теории банаховых пространств (см. [12, гл. 6, §3 и $§ 9$, упр. 18]), для одномерного подпространства $M_{x}$ в В существует дополнение - замкнутое подпространство $N_{x}$ такое, что верно представление $\mathbf{B}=M_{x} \oplus N_{x}$. Это означает, что каждый элемент $y \in \mathbf{B}$ может быть единственным образом представлен в виде суммы $y=y_{1}+y_{2}$, где $y_{1} \in M_{x}, y_{2} \in N_{x}$. При этом существует проектор $\Pi_{M_{x}}$ на подпространство $M_{x}$ такой, что $M_{x}=\Pi_{M_{x}} \mathbf{B}, N_{x}=\Pi_{N_{x}} \mathbf{B}$, здесь $\Pi_{N_{x}}:=\mathscr{I}-\Pi_{M_{x}}$ является оператором проектирования на подпространство $N_{x}$.

Очевидно, что $\widehat{N}_{x}:=N_{x}+x$ есть аффинная гиперплоскость коразмерности 1 , проходящая через точку $x$.

(B4) Функиия $Q$ дважды дифферениируема по Фреше в некоторой окрестности $U_{0}$ многообразиия $M$, принадлежит классу $\operatorname{Hol}_{\alpha}^{2}\left(U_{0}\right)$ для некоторого $\alpha>0$ и удовлетворяет условию невырожденности: для всех $x \in M$ имеет место неравенство

$$
\left\langle y,\left[A^{-1}-\lambda Q^{\prime \prime}(x)\right] y\right\rangle>0, \quad 0 \neq y \in N_{x} \cap \operatorname{Dom}\left(A^{-1}\right) \cap \operatorname{Dom}\left(Q^{\prime \prime}(x)\right),
$$

где $\lambda=\left\langle x, A^{-1} x\right\rangle /\left\langle x, Q^{\prime}(x)\right\rangle>0$ (множитель Лагранжа, см. лемму 1 в разделе 2).

Пересечение $S_{x}:=\widehat{N}_{x} \cap \partial D$ является гладкой поверхностью коразмерности 2, которую можно задать в виде

$$
S_{x}=\left\{y \in \widehat{N}_{x}: F(y)=0\right\},
$$

здесь, в силу условия (В4), функция $F(y)$ дважды дифференцируема по Фреше в окрестности $U_{0} \cap \widehat{N}_{x}$. В наших условиях, вследствие формул (1.3), (1.5), функция $F(y)$ определяется единственным образом из 
уравнения

$$
S_{x}=\left\{y \in \mathbf{B}: Q(y)=0, y-x \in N_{x}\right\} .
$$

Напомним (см. лемму 3 из $[12$, гл. $6, \S 3]$ ), что оператор $\Pi_{M_{x}}^{*}$, сопряженный к проектору $\Pi_{M_{x}}$, является проекционным оператором в банаховом пространстве $\mathbf{B}^{*}$, причем

$$
\Pi_{M_{x}}^{*} \mathbf{B}^{*}=\left\{y^{*}:\left\langle y, y^{*}\right\rangle=0 \text { для всех } y \in N_{x}\right\} .
$$

Последнее множество есть одномерное подпространство в $\mathbf{B}^{*}$, поэтому мы можем отождествить его с пространством $M_{x}^{*}-$ сопряженным пространством одномерного подпространства $M_{x}$. Таким образом,

$$
M_{x}^{*} \equiv \Pi_{M_{x}}^{*} \mathbf{B}^{*}=\left\{y^{*}:\left\langle y, y^{*}\right\rangle=0 \text { для всех } y \in N_{x}\right\} .
$$

Отсюда и из леммы 3 книги [12, гл. $6, \S 3]$ вытекает, что

$$
N_{x}^{*} \equiv \Pi_{N_{x}}^{*} \mathbf{B}^{*}=\left\{y^{*}:\left\langle n_{x}, y^{*}\right\rangle=0\right\},
$$

где $\Pi_{N_{x}}^{*}:=\mathscr{I}-\Pi_{M_{x}}^{*}$ является оператором проектирования на подпространство $N_{x}^{*}$. Следовательно, $\Pi_{M_{x}}^{*} \equiv \Pi_{M_{x}^{*}}, \Pi_{N_{x}}^{*} \equiv \Pi_{N_{x}^{*}}$.

Мы будем рассматривать подпространство $N_{x}$ как банахово пространство с нормой $\|\cdot\|$, при этом определенное выше подпространство $N_{x}^{*}$ является его сопряженным пространством, двойственность между $N_{x}$ и $N_{x}^{*}$ будем по-прежнему обозначать через $\langle\cdot, \cdot\rangle$.

Определим оператор $F^{\prime \prime}(x): N_{x} \rightarrow N_{x}^{*}$. Введем функцию $\widetilde{F}(z):=$ $F(z+x), z \in N_{x}$. В силу наложенных условий, существует вторая производная по Фреше $\widetilde{F}^{\prime \prime}(0)=F^{\prime \prime}(x)$, которую мы можем рассматривать как линейный оператор, действующий из $N_{x}$ в $N_{x}^{*}$.

Введем также линейный оператор $A^{N_{x}}:=\Pi_{N_{x}} A \Pi_{N_{x}}^{*}$. Область значений этого оператора есть пространство $N_{x}$. Пусть $n_{x}^{*} \in \mathbf{B}^{*}$ обозначает единичный вектор, на который натянуто одномерное пространство $M_{x}^{*}$.

Теорема 1. Пусть выполнень сформулированнье выше предположения, включая условия (B1)-(B4). Тогда при $u \rightarrow \infty$ справедливо асимптотическое соотношение

$$
P_{A}(u D)=\exp \left\{-\frac{u^{2}}{2 \sigma^{2}}\right\} \frac{1}{2 \pi} \int_{M} c(x) d V_{M}(x)(1+o(1)),
$$

где

$$
\begin{aligned}
c(x):= & {\left[\left\langle A n_{x}^{*}, n_{x}^{*}\right\rangle\left|\operatorname{det} B_{x}\left\langle B_{x}^{-1} A^{N_{x}} a_{x}, a_{x}\right\rangle\right|\right]^{-1 / 2}, } \\
& B_{x}:=\mathscr{I}-\lambda A^{N_{x}} F^{\prime \prime}(x)
\end{aligned}
$$

- линейный оператор, отображающий $N_{x}$ в $N_{x}$,

$$
a_{x}:=A^{-1} x,
$$


$u d V_{M}$ - элемент длинь на кривой $M$, справедливо при условии, что конечен детерминант оператора $B_{x}$ :

$$
\operatorname{det} B_{x}:=\prod_{k=1}^{\infty}\left(1-\beta_{k}\right)
$$

здесь $\beta_{k}, k=1,2 \ldots$, являются собственными значениями оператора $\lambda A^{N_{x}} F^{\prime \prime}(x): N_{x} \rightarrow N_{x}$.

Заметим, что в формуле (1.8) в качестве элемента $a_{x}$ можно брать $\Pi_{N_{x}^{*}} A^{-1} x$. Напомним (см. $[27$, гл. 27]), что для ядерного оператора $C$ в банаховом пространстве $B$ определен детерминант

$$
\operatorname{det}(\mathscr{I}+C):=\prod_{k=1}^{\infty}\left(1+\nu_{k}\right)
$$

где $\left\{\nu_{k}\right\}-$ последовательность собственных чисел оператора $C$. Для ядерных операторов в хороших пространствах (например, в пространствах $L^{p}$ ) произведение (1.11), как правило, конечно см. [32], [34], [36]. Довольно сложный вопрос о конечности произведения (1.11) в случае ядерных операторов в общих банаховых пространствах рассмотрен в книгах [27], [56] (см. также замечание 1 в [34]).

Если оператор $C: B \rightarrow B$ может быть записан в матричном виде $C=\left(c_{i j}\right)_{i, j=1, \infty}$ (например, относительно некоторого базиса Шаудера), то для вычисления детерминанта (1.11) можно брать конечномерные аппроксимации:

$$
\operatorname{det}(\mathscr{I}+C)=\lim _{k \rightarrow \infty} \operatorname{det}\left(\mathscr{I}+C_{k}\right)
$$

где $C_{k}=\left(c_{i j}\right)_{i, j=\overline{1, k}}$. Свойства детерминантов операторов $\mathscr{I}+C$ в гильбертовом пространстве приведены в книге [11].

3 а м е ч а н и е 1 . Пусть любые элементы $y$ и $y^{*}$ банаховых пространств $\mathbf{B}$ и $\mathbf{B}^{*}$ допускают представление в виде последовательностей

$$
y=\left(y_{1}, y_{2}, \ldots, y_{k}, \ldots\right) \in \mathbf{B}, \quad y^{*}=\left(y_{1}^{*}, y_{2}^{*}, \ldots, y_{k}^{*}, \ldots\right) \in \mathbf{B}^{*},
$$

а рассматриваемые линейные операторы допускают представление в виде бесконечных матриц. Такая ситуация естественно возникает в двух случаях. Первый, общеизвестный случай - когда в обоих пространствах $\mathbf{B}$ и $\mathbf{B}^{*}$ существуют базисы Шаудера (см. [15, гл. $13, \S 7$, п. 7.2], $[18$, гл. $1, \S 6])$. Второй, менее известный случай - когда осуществляется переход от исходной гауссовской меры $P_{A}$ на $\mathbf{B}$ к ее эквивалентному варианту - гауссовской мере $P_{\widehat{A}}$, заданной на банаховом пространстве последовательностей $\widehat{\mathbf{B}}$ (см. [26, $\S 3.3])$.

При таком подходе, если выполнены условия $n_{x}^{*}=(1,0, \ldots, 0, \ldots)$, $x=\left(0, x_{2}, \ldots, x_{n}, \ldots\right)$, то для вычислений может оказаться более удобной следующая формула для функции $c(x)$ :

$$
c(x)=|\operatorname{det} \Lambda(x)|^{-1 / 2}, \quad x \in M,
$$


где $\Lambda(x)$ - линейный оператор, имеющий матричное представление

$$
\Lambda(x)=\left[\begin{array}{ccc}
0 & 0 & A^{-1} x \\
0 & 0 & n_{x}^{*} \\
A n_{x}^{*} & x & \mathscr{I}-\lambda A Q^{\prime \prime}(x)
\end{array}\right] .
$$

Формула (1.13) понимается в следующем координатном смысле. Элементы $A^{-1} x \in \mathbf{B}^{*}, n_{x}^{*} \in \mathbf{B}^{*}$, записываются в виде вектор-строки своих координат, элементы $A n_{x}^{*} \in \mathbf{B}, x \in \mathbf{B},-$ в виде вектор-столбцов, а оператор $\mathscr{I}-\lambda A Q^{\prime \prime}(x): \mathbf{B} \rightarrow \mathbf{B}-$ в матричном виде. Матрица $\Lambda(x)$ состоит из этих блоков и двумерной нулевой матрицы, стоящей в верхнем левом углу.

Для упрощения вычислений по формулам (1.12), (1.13) удобно брать матричное представление оператора $\mathscr{I}-\lambda A Q^{\prime \prime}(x)$ в диагональном виде.

3 а м е ч а н и е 2 . Довольно часто оператор $A$, множества $D$ и $M$ обладают некоторыми свойствами симметрии (подробное определение для случая гильбертова пространства имеется в [47, ч. II, с. 156]). В такой ситуации значение функции $c(x)$ равно одному и тому же числу $c$ для всех точек $x \in M$ и $\int_{M} c(x) d V_{M}(x)=c \operatorname{mes}(M)$, где mes $(M)-$ длина кривой $M$. Два подобных примера даны ниже, в следствии 1.

3 а м е ч а н и е 3 . Условие дифференцируемости функционала $Q(x)$ из (B4) можно заменить на следующее, более слабое. Пусть существуют окрестность $U_{1}$ многообразиия $M$ и всюду плотное множество $T \subset U_{1}$ такие, что функция $Q$ дважды дифференцируема по Фреше в точках множества $T$ и $Q$ принадлежит классу $\operatorname{Hol}_{\alpha}^{2}(T)$ для некоторого $\alpha>0$. Напомним, что в определении производной по Фреше, данном в $[17, \S 2$, п. 2.2$]$, допускается, чтобы рассматриваемый оператор был определен лишь на некотором всюду плотном подмножестве банахова пространства.

П р и м е р. Точные асимптотики больших уклонений распределений $L^{p}$-функционалов для центрированного броуновского моста, $p>0$. $L^{p}$-функционалом мы называем функционал

$$
G(x):=\int_{0}^{1}|x(t)|^{p} d t, \quad p>0,
$$

определенный на том или ином пространстве функций на $[0,1]$. Пусть $w(t)$ - стандартный винеровский процесс и $w_{0}(t)=w(t)-t w(1), t \in$ $[0,1],-$ броуновский мост. Определим гауссовский процесс

$$
\xi(t):=w_{0}(t)-\int_{0}^{1} w_{0}(s) d s, \quad t \in[0,1]
$$

который называется центрированным броуновским мостом (см. [57]). Этот процесс возникает в качестве слабого предела эмпирического процесса при рассмотрении статистики Ватсона (см. [21, гл. 2, §9], [24, гл. $2, \S 2.1, \S 2.5],[44],[49],[62])$. 
Гауссовский процесс $\xi(t)$ имеет п.н. непрерывные траектории, среднее нуль и ковариационную функцию

$$
r(t, s):=\frac{1}{2}\left[(t-s)^{2}-|t-s|+\frac{1}{6}\right], \quad t, s \in[0,1] .
$$

Очевидно, что $\int_{0}^{1} \xi(t) d t=0$. Пусть $\mu \equiv \mu_{\xi}$ обозначает распределение процесса $\xi(t)$ в пространстве $C[0,1]$ непрерывных на $[0,1]$ функций, снабженном нормой супремума (см. [9, гл. 5], [10], [20]). Применяя теорему 1 , получаем следующий результат.

На протяжении всей статьи $\Gamma(\cdot)$ обозначает гамма-функцию.

Теорема 2. Для $0<p \leqslant 6$ при $u \rightarrow \infty$ выполнено соотношение

$$
\begin{aligned}
\mathbf{P}\left\{\int_{0}^{1}|\xi(t)|^{p} d t>u^{p}\right\} & =\mu_{\xi}\left\{\int_{0}^{1}|x(t)|^{p} d t>u^{p}\right\} \\
& =\exp \left\{-\frac{u^{2}}{2 \sigma^{2}(p)}\right\} \frac{K(p)}{2 \pi}(1+o(1)),
\end{aligned}
$$

гдe

$$
\sigma^{-2}(p)=\frac{8 \pi}{p}\left(1+\frac{p}{2}\right)^{(2-p) / p}\left[\frac{\Gamma(1 / p)}{\Gamma(1 / 2+1 / p)}\right]^{2}
$$

$0<K(p)<\infty$.

Константу $K(p)$ можно вычислять по формулам (1.7), (1.12), (1.13) с $M=M_{0}$, где $M_{0}$ определено ниже в соотношении (3.14).

Ограничение сверху на $p$ в теореме 2 вытекает из того факта, что множество точек минимума из условия (В3) при $0<p \leqslant 6$ качественно отличается от множества точек минимума из этого условия при $p>6$ (см. пример 1 в статье [55] и замечание 4 в разделе 3 ниже).

В двух наиболее простых случаях, при $p=1$ и $p=2$, найдены точные значения константы $K(p)$.

Следствие 1. При $u \rightarrow \infty$ выполнены соотношения

$$
\begin{aligned}
\mathbf{P}\left\{\int_{0}^{1}|\xi(t)| d t>u\right\} & =\mu_{\xi}\left\{\int_{0}^{1}|x(t)| d t>u\right\} \\
& =\exp \left\{-24 u^{2}\right\}(1+o(1)) \\
\mathbf{P}\left\{\int_{0}^{1} \xi^{2}(t) d t>u^{2}\right\} & =\mu_{\xi}\left\{\int_{0}^{1} x^{2}(t) d t>u^{2}\right\} \\
& =2 \exp \left\{-2 \pi^{2} u^{2}\right\}(1+o(1)) .
\end{aligned}
$$

Логарифмическая асимптотика вероятности из формулы (1.18) была ранее вычислена в работе [57], показатель $2 \pi^{2}$ был найден в статье [62] (см. также [24, с. 75]). Соотношение (1.19) можно получить также еще двумя разными способами: применяя последнюю формулу из 9 главы 2 книги $[21$, с. 75$]$ и используя формулу В. М. Золотарева [13], [26, §3] (см. теорему 3 в п. 4.2). 
2. Основные положения метода Лапласа. Доказательство теоремы 1. Изложим основные положения метода Лапласа для гауссовских мер, следуя той же схеме, что и в работах $[26, \S 3.3],[32, \S 2]$, и уделяя при этом особое внимание условию (B3). Мы также будем учитывать результаты статьи [47, ч. II], где исследована точная асимптотика гауссовского интеграла $J_{u}(H)$ (см. (1.1)) в случае, когда функционал действия имеет конечномерное многообразие точек минимума. В [47, ч. II] подробно рассмотрен случай гильбертова пространства $\mathbf{B} \equiv H$, основной результат доказан также и для банахова пространства $C[0,1]$ (см. [47, ч. II, §6, теорема 5]). Метод работы [47, ч. II] допускает обобщение на общие банаховы пространства (см. также [41, ч. II, §6]), поэтому мы будем использовать некоторые утверждения из [47, ч. II], справедливые и в нашем случае гауссовской меры $P_{A}$ в пространстве $\mathbf{B}$.

Пусть $\left(H_{A},\|\cdot\|_{A}\right)$ обозначает гильбертово пространство, ассоциированное с оператором $A$, т.е. пополнение области значений $R(A)$ оператора $A$ относительно предгильбертовой нормы $\|A y\|_{A}:=\sqrt{\langle A y, y\rangle}$, $y \in \mathbf{B}^{*}$ (см. предложение 1.6 в $[6$, гл. 3$\left.]\right)$.

Определим функционал действия гауссовской меры $P_{A}$ следующим образом:

$$
I(x)= \begin{cases}\frac{1}{2}\|x\|_{A}^{2}, & \text { если } x \in H_{A} \\ +\infty & \text { в противном случае. }\end{cases}
$$

Отсюда видно, что $I(x)$, рассматриваемая как функция на $H_{A}$, бесконечно дифференцируема по Фреше на $\left(H_{A},\|\cdot\|_{A}\right)$. Однако $I(x)$ недифференцируема на $(H,\|\cdot\|)$. Свойства функционала $I(x)$ описаны, например, в работе $[26, \S 2]$. Отметим, что

$$
I(x)=\frac{1}{2}\left\langle x, A^{-1} x\right\rangle, \quad x \in R(A) .
$$

Перейдем к изложению основных лемм метода Лапласа, полагая выполненными условия (B1)-(B4).

Лемма 1 (решение экстремальной задачи в $D$ ). (i) Функиионал действия $I(x)$ достигает своего минимума на множестве $D$ на одномерной кривой $M$, причем для каждой точки $m \in M$ выполнены равенства

$$
\begin{gathered}
Q(m)=0, \\
A^{-1} m-\lambda Q^{\prime}(m)=0,
\end{gathered}
$$

где $\lambda>0$ - иисло из условия (B4).

(ii) Для каждой точки $m \in M$ имеет место неравенство (условие второго порядка)

$$
\left\langle y,\left[A^{-1}-\lambda Q^{\prime \prime}(m)\right] y\right\rangle \geqslant 0, \quad 0 \neq y \in N_{m} \cap \operatorname{Dom}\left(A^{-1}\right) \cap \operatorname{Dom}\left(Q^{\prime \prime}(m)\right) .
$$


Д о к а з а т е л ь с т в леммы проводится при помощи метода множителей Лагранжа $[2$, гл. 3 , пп. 3.2.1, 3.4.1], дающего необходимые условия (первого и второго порядка) минимума функции $I(x)$ при ограничении $Q(x) \geqslant 0$. В случае, когда множество минимума $M$ состоит из одной точки $x^{0}$, аналог леммы 1 подробно доказан в $[32, \S 2$, лемма 1$]$. Наш случай одномерного многообразия $M$ рассматривается совершенно аналогично.

Отметим, что в условии невырожденности из (В4) мы требуем выполнения строгого неравенства из утверждения (ii) леммы 1.

Лемма 2 (о логарифмической асимптотике). При выполнении условий (B1)-(В3) имеют место соотношения

$$
\begin{aligned}
\lim _{u \rightarrow \infty} \frac{1}{u^{2}} \ln P_{A}(u D) & =-\inf _{x \in D} I(x)=-\frac{1}{2}\left\langle m, A^{-1} m\right\rangle \\
& =-\sigma^{-2} \quad \text { для всех } m \in M .
\end{aligned}
$$

Первое, наиболее важное, соотношение в формуле (2.5) доказано в $[43$, c. 34 , утверждение (iv)], оно имеет в своей основе уже упоминавшийся фундаментальный принцип больших уклонений для гауссовских мер в банаховых пространствах (см. также [26, пример 2.4]). Два последних равенства в формуле (2.5) следуют непосредственно из леммы 1 и соотношений $(1.4),(2.2)$.

Отметим, что условие выпуклости из (В2) существенно для справедливости леммы 2. Лемма 2 составляет основу метода Лапласа и позволяет ограничиться исследованием асимптотики гауссовской меры малой полуокрестности множества $M$. Следуя [47, ч. II, с. 161,175$]$, для $\delta>0$ введем трубчатую окрестность $M(\delta)$ множества $M$ :

$$
M(\delta):=\left\{x \in \mathbf{B}: \inf _{m \in M}\|x-m\|<\delta\right\},
$$

и положим

$$
\mathscr{V}=\mathscr{V}(\delta):=M(\delta) \cap D=\left\{x \in D: \inf _{m \in M}\|x-m\|<\delta\right\} .
$$

Как указано в [47, ч. II, с. 161,176$]$, для достаточно малых $\delta<\delta_{0}$ трубчатая окрестность $M(\delta)$ обладает рядом хороших свойств, в частности,

каждая точка $y \in M(\delta)$ может быть записана единственным

образом в виде $y=x+z$, где $x \in M, z \in \widehat{N}_{x},\|z\|<\delta$.

Ниже мы считаем, что $\delta$ выбрано достаточно малым.

Лемма 3 (о выделении малой полуокрестности множества минимума). Для любого $\delta>0$ найдется такое $\kappa=\kappa(\delta)>0$, ито при $u \rightarrow \infty$ имеет место соотношение

$$
P_{A}(u D)=P_{A}(u \mathscr{V})\left(1+O\left(e^{-u^{2} \kappa}\right)\right) .
$$


Д о к а з а т е л ь с т в о леммы стандартно; оно основано на лемме 2 и полунепрерывности снизу функционала действия $I(x)$ и проводится так же, как доказательство леммы 3 в [32], где рассмотрен случай одноточечного множества $M$. Соотношением, аналогичным $(2.8)$, является формула (3.5) из [47, ч. II, с. 162].

Для простоты дальнейшего изложения мы будем считать без ограничения общности, что многообразие $M$ представляет собой кривую

$$
M=\left\{x_{s}: 0 \leqslant s \leqslant \ell\right\}
$$

на поверхности $\partial D$, причем в качестве параметра используется длина дуги.

Согласно свойству (2.7) трубчатой окрестности, для точек $y=x+$ $z \in M(\delta), x=x_{s}$, определена функция

$$
I_{M}(x, z):=1-\left\langle\frac{d^{2} x_{s}}{d s^{2}}, z\right\rangle, \quad z \in \widehat{N}_{x}
$$

здесь, напомним, $\left\langle d^{2} x_{s} / d s^{2}, z\right\rangle-$ кривизна кривой $M$ в направлении $z$ (см. [47, ч. II, с. 156]).

Положим

$$
\widehat{R}_{x}:=\widehat{N}_{x} \cap \mathscr{V}(\delta), \quad R_{x}:=\widehat{R}_{x}-x, \quad x \in M,
$$

таким образом, $R_{x}$ - это малая полуокрестность нуля в подпространстве $N_{x}$. Пусть $P_{N_{x}}$ обозначает гауссовскую меру на подпространстве $N_{x}$ со средним нуль и ковариационным оператором $A^{N_{x}}, x \in M$.

Лемма 4. Для любого $и>0$ справедливо равенство

$$
\begin{aligned}
& \int_{\mathscr{V}} d P_{A}(u y)= \frac{u}{\sqrt{2 \pi}} \exp \left\{-\frac{u^{2}}{2 \sigma^{2}}\right\} \\
& \times \int_{M}\left[\int_{R_{x}} I_{M}(x, z) \exp \left\{-u^{2}\left\langle z, A^{-1} x\right\rangle\right\} d P_{N_{x}}(u z)\right] \\
& \times\left[\left\langle A n_{x}^{*}, n_{x}^{*}\right\rangle\right]^{-1 / 2} d V_{M}(x) .
\end{aligned}
$$

Утверждение леммы 4 представляет собой переформулировку в наших условиях важной леммы 3 из [47, ч. II, с. 162], которую можно записать в виде

$$
\begin{aligned}
\int_{\mathscr{V}} d P_{A}(u y)=\frac{1}{\sqrt{2 \pi}} \int_{M} & {\left[\int_{R_{x}} I_{M}(x, z)\right.} \\
\times & \left.\exp \left\{-\frac{u^{2}}{2}\left\langle x, A^{-1} x\right\rangle-u^{2}\left\langle z, A^{-1} x\right\rangle\right\} d P_{N_{x}}(u z)\right] \\
\times & {\left[\left\langle A n_{x}^{*}, n_{x}^{*}\right\rangle\right]^{-1 / 2} d V_{M}(u x) . }
\end{aligned}
$$


Формула (2.13) представляет собой некоторое обобщение известной формулы Камерона-Мартина о сдвиге гауссовской меры (ср. с леммой 3.7 из [26] в случае одноточечного множества $M)$. Из (2.13) легко получаем (2.12), учитывая, что $d V_{M}(u x)=u d V_{M}(x)$. Отметим, что одномерная гауссовская мера $A_{M_{x}}$ из работы $[47$, ч. II], заданная на подпространстве $M_{x}$, в нашем случае является одномерной гауссовской мерой со средним нуль и дисперсией $\left\langle A n_{x}^{*}, n_{x}^{*}\right\rangle$.

Лемма 5 (о точной асимптотике внутреннего интеграла в (2.12)). При $u \rightarrow \infty$ следующее асимптотическое соотношение выполнено равномерно по $x \in M$ :

$$
\begin{aligned}
T_{u}(x) & :=\int_{R_{x}} I_{M}(x, z) \exp \left\{-u^{2}\left\langle z, A^{-1} x\right\rangle\right\} d P_{N_{x}}(u z) \\
& =\frac{1}{u \sqrt{2 \pi}}\left[\left|\operatorname{det} B_{x}\left\langle B_{x}^{-1} A^{N_{x}} a_{x}, a_{x}\right\rangle\right|\right]^{-1 / 2}(1+o(1)),
\end{aligned}
$$

здесь использовань обозначения (1.9), (1.10).

Д ок а з а т е ль с т в о. Несложно убедиться, что для фиксированного $x \in M$ к интегралу $T_{u}(x)$, взятому по малой полуокрестности нуля $R_{x}$ в банаховом пространстве $N_{x}$, применима теорема 3.4 из [26].

В самом деле, пусть функционал действия $I_{0}(z)$ гауссовской меры $P_{N_{x}}$ определяется формулой $(2.1)$, записанной для пространства $N_{x}$. Евклидово действие $G_{0}(z)$, определенное в $[26$, формула $(3.2)]$, в нашем случае имеет вид

$$
G_{0}(z):=\left\langle z, A^{-1} x\right\rangle+I_{0}(z), \quad z \in N_{x} .
$$

Покажем, что функция $G_{0}(z)$ достигает своего минимума на множестве $R_{x}$ в единственной точке $z=0$ и при этом $G_{0}(0)=0$.

Из формул $(2.6),(2.7),(2.11)$ следует, что для точки $z \in R_{x}$ выполнено неравенство $Q(z+x) \geqslant 0,\|z\|<\delta$. Используя разложение Тейлора гладкой функции $Q(z+x)$ в окрестности точки $x$ (см. [2, п. 2.2.5]), получаем:

$$
0 \leqslant Q(z+x)=Q(x)+\left\langle z, Q^{\prime}(x)\right\rangle+O\left(\|z\|^{2}\right), \quad z \in R_{x} .
$$

Отсюда, с учетом соотношений $(2.3),(2.4)$ и того, что $\lambda>0$, приходим к неравенству

$$
\left\langle z, A^{-1} x\right\rangle \geqslant 0, \quad z \in R_{x} .
$$

Поскольку $I_{0}(z)=0$ тогда и только тогда, когда $z=0$, то из $(2.15),(2.16)$ следует сформулированное выше утверждение о единственности точке минимума функции $G_{0}(z)$. Применяя формулу (3.12) из [26], получаем соотношение (2.14) для фиксированного $x \in M$, так как, согласно (2.10), $I_{M}(x, 0)=1$. 
Утверждение о равномерности по $x \in M$ асимптотического соотношения (2.14) получается посредством аккуратного оценивания остаточных членов по схеме работ [47, ч. I, с. 460], [47, ч. II, с. 164], с учетом компактности множества $M$ и условия типа Гёльдера из (B4). Лемма 5 доказана.

Д ок а з а т е л ь с т в о т е о р е м ы 1 . Поскольку асимптотическое соотношение (2.14) выполнено равномерно по $x \in M$, то допустимо его почленное интегрирование, следовательно, из формул (2.12) и (2.14) получаем

$$
P_{A}(u \mathscr{V})=\exp \left\{-\frac{u^{2}}{2 \sigma^{2}}\right\} \frac{1}{2 \pi} \int_{M} c(x) d V_{M}(x)(1+o(1)), \quad u \rightarrow \infty .
$$

Отсюда, в силу (2.8) вытекает искомая формула (1.7). Теорема 1 доказана.

Доказательство замечания 1 основано на координатной записи формулы (1.8), конечномерных аппроксимациях и достаточно элементарных преобразованиях возникающих конечномерных определителей. Это доказательство довольно длинное и потому здесь опущено.

Приведем один полезный результат, который нам понадобится ниже.

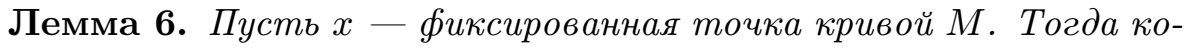
вариачионньй оператор $A^{N_{x}}$ гауссовской мерь $P_{N_{x}}$ имеет вид

$$
A^{N_{x}} z=A z-\frac{\left\langle A n_{x}^{*}, z\right\rangle}{\left\langle A n_{x}^{*}, n_{x}^{*}\right\rangle} A n_{x}^{*}, \quad z \in N_{x}^{*}
$$

Д о к а з а т е л ь с т в о леммы имеется в [32, лемма 6].

3. Большие уклонения распределений $L^{p}$-функционалов для центрированного броуновского моста. Доказательство теоремы 2. Пусть $L^{p}=L^{p}[0,1], p \geqslant 1$, - обычное банахово пространство функций $x(t)$ на $[0,1]$ с нормой

$$
\|x\|_{L^{p}}:=[G(x)]^{1 / p} \equiv\left(\int_{0}^{1}|x(t)|^{p} d t\right)^{1 / p} .
$$

Для $0<p<1$ обозначим через $L^{p}$ множество измеримых функций $x(t)$ на $[0,1]$, для которых конечен интеграл $G(x) \equiv \int_{0}^{1}|x(t)|^{p} d t$ (см. $[17$, $\S 1$, п. 1.1]). Используя неравенство Гёльдера $[17, \S 1$, п. 1.1], несложно получить включение: $L^{r_{2}} \subset L^{r_{1}}$, если $0<r_{1}<r_{2}<\infty$.

При работе с $L^{p}$-функционалом (1.14) выбор подходящего банахова функционального пространства, на котором удобно рассматривать гауссовскую меру $\mu_{\xi}$, следует проводить в зависимости от того, будут ли выполнены неравенства $p>1 / 2$, или $0<p \leqslant 1 / 2$. 
Положим

$$
\widetilde{p}:= \begin{cases}2, & \text { если } p>\frac{1}{2}, \\ \frac{1}{p}+\varepsilon_{0}, & \text { если } 0<p \leqslant \frac{1}{2},\end{cases}
$$

где $\varepsilon_{0}>0$ - любое фиксированное число. Пусть $\widetilde{q}>1$ обозначает число, сопряженное к $\widetilde{p}>1$, т.е. $1 / \widetilde{p}+1 / \widetilde{q}=1$.

Для числа $a>1$ определим

$$
L_{0}^{a}:=\left\{x \in L^{a}: \int_{0}^{1} x(t) d t=0\right\}
$$

- собственное подпространство пространства $L^{a}$, и пусть $C_{0}[0,1]:=$ $\left\{x \in C[0,1]: \int_{0}^{1} x(t) d t=0\right\}$.

Мы будем рассматривать гауссовскую меру $\mu_{\xi}$, считая ее заданной в пространстве $L_{0}^{\tilde{p}}$, имея в виду, что эта мера сосредоточена на $C_{0}[0,1]$.

В этом случае мера $\mu_{\xi}$ имеет нулевое среднее и инъективный ковариационный оператор $A=A_{\mu}: L_{0}^{\tilde{q}} \rightarrow L_{0}^{\tilde{p}}$ интегрального типа:

$$
A x(t)=\int_{0}^{1} r(t, s) x(s) d s, \quad x \in L_{0}^{\tilde{q}},
$$

где $r(t, s)$ задана формулой (1.15).

Будем полагать, что функционал $G$ из $(1.14)$ определен на $L_{0}^{\tilde{p}}$, и возьмем в качестве множества $D$ множество

$$
D=\left\{x \in L_{0}^{\tilde{p}}: G(x) \geqslant 1\right\}
$$

Пусть $C_{0}^{2} \equiv C_{0}^{2}[0,1]$ - множество функций из $C_{0}[0,1]$, дважды дифференцируемых всюду на отрезке $[0,1]$.

Лемма 7. Область значений ковариачионного оператора $A_{\mu}$, определенного формулой (3.4), имеет вид

$$
R\left(A_{\mu}\right)=\left\{x \in C_{0}^{2}: x(0)=x(1), x^{\prime}(0)=x^{\prime}(1)\right\},
$$

для $y \in R\left(A_{\mu}\right)$ обратный оператор задается формулой

$$
\left[A_{\mu}^{-1} y\right](t)=-y^{\prime \prime}(t) \quad \text { для всех } t \in[0,1] .
$$

Д ок аз а т ель с тв о. Утверждения леммы получаются в результате двукратного дифференцирования по $t$ равенства

$$
y(t)=A x(t) \equiv \frac{1}{2} \int_{0}^{1}\left[(t-s)^{2}-|t-s|+\frac{1}{6}\right] x(s) d s, \quad x \in L_{0}^{\tilde{q}} .
$$

Отметим, что формулы (3.6), (3.7) фактически доказаны также в [57, c. 5]. Укажем, что условия типа равенств в формуле (3.6) представляют собой периодические граничные условия. 
Решение экстремальной задачи. Для $0<p \leqslant 6$ определим числа

$$
C_{0}:=1+\frac{p}{2}, \quad \lambda_{0}:=\frac{1}{p \sigma^{2}(p)}=\frac{8 \pi}{p^{2}}\left(1+\frac{p}{2}\right)^{2 / p-1}\left[\frac{\Gamma(1 / p)}{\Gamma(1 / p+1 / 2)}\right]^{2},
$$

здесь величина $\sigma^{2}(p)$ задана формулой (1.17).

Пусть $F(\cdot, \cdot ; \cdot ; z)$ обозначает гипергеометрическую функцию (см. [1, гл. 15], [3, гл. 2]). Зададим функцию $x_{0}(t)$ на отрезке $[0,1]$ следующим образом.

На отрезке $[0,1 / 4]$ функиия $x_{0}(t)$ определяется как единственное положительное решение уравнения

$$
x(t) F\left(\frac{1}{2}, \frac{1}{p} ; 1+\frac{1}{p} ; \frac{x^{p}(t)}{C_{0}}\right)=\sqrt{2 \lambda_{0} C_{0}} t, \quad t \in\left[0, \frac{1}{4}\right] .
$$

Уравнение типа (3.9) ранее было получено и исследовано в [34, п. 3], [32, п. 4.1]. Известно, что $x_{0}(0)=0$, функция $x_{0}(t)$ дважды непрерывно дифференцируема, строго возрастает на отрезке $[0,1 / 4]$, достигает своего максимума в точке $t=1 / 4, x_{0}(1 / 4)=C_{0}^{1 / p}$ и при этом $x_{0}^{\prime}(1 / 4)=0$.

На отрезке $[1 / 4,1]$ функиия $x_{0}(t)$ определяется равенствами

$$
\begin{array}{ll}
x_{0}(t)=x_{0}\left(\frac{1}{2}-t\right), & t \in\left[\frac{1}{4}, \frac{1}{2}\right], \\
x_{0}(t)=-x_{0}(1-t), & t \in\left[\frac{1}{2}, 1\right] .
\end{array}
$$

График функции $x_{0}(t)$ на отрезке $[0,1]$ (похожий на график функции $\sin (2 \pi t)$, ср. с предложением 3 ниже) имеет центр симметрии в точке $t=1 / 2$, а на отрезке $[0,1 / 2]$ он имеет ось симметрии в точке $t=1 / 4$. Из определения (3.9)-(3.11) следует, что

$$
\int_{0}^{1} x_{0}(t) d t=0, \quad x_{0}(0)=x_{0}\left(\frac{1}{2}\right)=x_{0}(1)=0, \quad x_{0}^{\prime}(0)=x_{0}^{\prime}(1) .
$$

Таким образом, согласно формулам (3.6) и (3.12), функции $x_{0}$ принадлежит $R\left(A_{\mu}\right)$. Отметим, что $\left|x_{0}(t)\right| \leqslant C_{0}^{1 / p}, t \in[0,1]$. Учитывая соотношения (3.12), продолжим периодически функиию $x_{0}(t)$ на всю ось $\mathbf{R}$, полученную периодическую функиию с периодом 1 обозначим через $\widehat{x}_{0}(t), t \in \mathbf{R}$.

Для числа $0 \leqslant \gamma \leqslant 1$ определим функцию

$$
\widehat{x}_{\gamma}(t):=\widehat{x}_{0}(t-\gamma), \quad t \in \mathbf{R},
$$

- сдвиг функции $\widehat{x}_{0}(t)$ на $\gamma$ вправо, и положим

$$
x_{\gamma}(t):=\widehat{x}_{\gamma}(t), \quad t \in[0,1] .
$$


Очевидно, что $x_{\gamma} \in R\left(A_{\mu}\right)$. Введем множество

$$
M_{0}:=\left\{x_{\gamma}: 0 \leqslant \gamma \leqslant 1\right\} \subset R\left(A_{\mu}\right)
$$

(cp. с (2.9)). Отметим, что в (3.14) элементы $x_{0}$ и $x_{1}$ отождествлены, так что многообразие $M_{0}$ является замкнутой компактной кривой.

Лемма 8. (i) При $t \rightarrow 0$ вьполнено соотношение

$$
x_{0}(t)=\sqrt{2 \lambda_{0} C_{0}} t(1+o(1)) .
$$

При любом $\gamma \in[0,1]$ справедливо равенство

$$
\int_{0}^{1} p\left|x_{\gamma}(t)\right|^{p-1} \operatorname{sign} x_{\gamma}(t) d t=0 .
$$

Д о к а з а т е л ь с т в о. Из определения гипергеометрической функции следует соотношение $F(1 / 2,1 / p ; 1+1 / p ; 0)=1$ (см. формулу 15.1 .1 в [1, гл. 15]). Отсюда и из формулы (3.9), с учетом равенства $x_{0}(0)=0$, получаем соотношение $(3.15)$.

Поскольку $p>0$, то в силу (3.15) функция $\left|x_{0}(t)\right|^{p-1}$ принадлежит $L^{1}$. При $\gamma=0$ равенство (3.16) получаем вследствие указанной выше симметрии функции $x_{0}(t)$. Для общего $0<\gamma<1$ равенство (3.16) следует из свойства периодичности, использованного в определении функции $x_{\gamma}$. Лемма 8 доказана.

Напомним, что множество $D$ определено формулой (3.5).

Лемма 9. При $0<p \leqslant 6$ минимум в экстремальной задаче

$$
\left\langle x, A_{\mu}^{-1} x\right\rangle \rightarrow \inf , \quad x \in D,
$$

равен $\sigma^{-2}(p)$ и достигается на одномерном дважды дифферениируемом компактном многообразии $M_{0}$, лежащем на граниче $\partial D$.

3 а м е ч а н и е 4. (i) Из результатов примера 1 статьи [55] и теоремы 1 работы [23] вытекает, что семейство экстремалей, доставляющих минимум в задаче (3.17) при $0<p \leqslant 6$, отличается от семейства экстремалей, доставляющих минимум в этой задаче при $p>6$. В частности, доказано, что при $0<p \leqslant 6$ среди экстремалей, доставляющих минимум в задаче (3.17), присутствует центрально-симметричная функция, в наших обозначениях, это функция $x_{0}(t)$.

(ii) В случае оператора (3.7), как правило, для широкого класса множеств $D, 0 \notin D$, минимум в экстремальной задаче типа (3.17) достигается на функциях, меняющих знак минимально возможное число раз. Это обстоятельство объясняется осцилляционной теоремой типа Штурма (см. [14, ч. 2, п. 9.2], [16, гл. 8]). В [32, п. 4.1], [34, §3] экстремальными были знакопостоянные функции. Учитывая, что теперь мы 
рассматриваем пространство функций $x(t)$, для которых $\int_{0}^{1} x(t) d t=0$, несложно убедиться, что среди функций, доставляющих минимум в экстремальной задаче (3.17), должна быть функция, имеющая ровно два нуля на интервале $[0,1)$ (ср. с теоремой 3.1 из [16, гл. 8]). Принимая во внимание это обстоятельство, а также упомянутые результаты из [23], [55], ниже мы особое внимание уделяем поиску и описанию минимизирующей центрально-симметричной функции с одной переменной знака.

Несложно видеть, что утверждение теоремы 2 следует из теоремы 1 и леммы 9 , формула (1.16) получается из соотношения (1.7).

Мы проведем доказательство леммы 9 в более сложном случае, когда $0<p \leqslant 1 / 2$. Более простой случай $p>1 / 2$ связан с гильбертовым пространством и исследуется аналогично. В частности, отметим, что, в силу (3.15), выполнено важное включение

$$
G^{\prime}\left(x_{0}\right)(t) \equiv p\left|x_{0}(t)\right|^{p-1} \operatorname{sign} x_{0}(t) \in L_{0}^{2}, \quad p>\frac{1}{2}
$$

(ср. с формулой (3.22) ниже).

Далее до кониа этого раздела мь полагаем, ито $0<p \leqslant 1 / 2$. Таким образом, согласно (3.2), $\widetilde{p}=1 / p+\varepsilon_{0}>\max (2,1 / p)$.

Для нас важно последнее неравенство. Легко проверить, что сопряженное число $\widetilde{q}$ удовлетворяет неравенству $1<\widetilde{q}<\min (2,1 /(1-p))$. Определим следующий класс функций:

$$
\mathscr{X}:=\left\{x \in C_{0}[0,1]: \frac{1}{x(t)} \in L_{0}^{\tilde{q}(1-p)}\right\} .
$$

Пусть также

$$
\mathscr{N}(x):=\{t \in[0,1]: x(t)=0\}, \quad x \in \mathscr{X} .
$$

Отметим, что множество $\mathscr{X}$ всюду плотно в $C_{0}[0,1]$, а следовательно, и в $L_{0}^{\tilde{p}}$. Очевидно также, что функция $y(t)$, для которой mes $\mathscr{N}(y)>0$, не принадлежит классу $\mathscr{X}$, здесь mes - мера Лебега. В частности, $0 \notin \mathscr{X}$.

Предложение 1. Функционал $G$ дважды дифферениируем по Фреше в каждой точке $x_{0} \in \mathscr{X}$ с производными

$$
\begin{aligned}
G^{\prime}\left(x_{0}\right)[h] & =\int_{0}^{1} p\left|x_{0}(t)\right|^{p-1} \operatorname{sign} x_{0}(t) h(t) d t, \quad h \in L_{0}^{\tilde{p}}, \\
G^{\prime \prime}\left(x_{0}\right)[h, g](t) & =\int_{0}^{1} p(p-1)\left|x_{0}(t)\right|^{p-2} h(t) g(t) d t, \quad h, g \in L_{0}^{\tilde{p}}
\end{aligned}
$$

Д о к а з а т е л ь с т в о. Утверждение предложения получаем на основе общих теорем о дифференцируемости интегральных функционалов в пространствах Лебега $L^{a}$ (см. $\left.[17, \S 20]\right)$. 
Как известно, каноническая билинейная форма между пространствами $L_{0}^{\tilde{p}}$ и $L_{0}^{\tilde{q}}$ имеет интегральный вид:

$$
\langle h, g\rangle=\int_{0}^{1} h(t) g(t) d t, \quad h \in L_{0}^{\tilde{p}}, \quad g \in L_{0}^{\tilde{q}} .
$$

3 а м е ч а н и е 5 . Пусть $x_{0} \in \mathscr{X}$. Тогда, в силу соотношений (3.19), (3.20), мы вправе использовать далее следующую форму записи: $G^{\prime}\left(x_{0}\right)[h]=\left\langle h, G^{\prime}\left(x_{0}\right)\right\rangle$, где справа стоит

$$
G^{\prime}\left(x_{0}\right)(t)=p\left|x_{0}(t)\right|^{p-1} \operatorname{sign} x_{0}(t)
$$

— элемент пространства $L_{0}^{\tilde{q}}$ (см. (3.18)), и

$$
G^{\prime \prime}\left(x_{0}\right)[h, g](t)=\left\langle h, G^{\prime \prime}\left(x_{0}\right) g\right\rangle, \quad h, g \in L_{0}^{\tilde{p}},
$$

справа в (3.23) стоит линейный оператор умножения $G^{\prime \prime}\left(x_{0}\right): L_{0}^{\tilde{p}} \rightarrow L_{0}^{\tilde{q}}$, действующий по правилу

$$
\left[G^{\prime \prime}\left(x_{0}\right) g\right](t)=p(p-1)\left|x_{0}(t)\right|^{p-2} g(t), \quad g \in L_{0}^{\tilde{p}} .
$$

Лемма 10. Для каждого $\gamma \in[0,1]$ функиия $x_{\gamma}$ принадлежит $\mathscr{X}$.

Д о к а з а т е л ь с т в о. Включение $x_{0} \in \mathscr{X}$ вытекает из определения функции $x_{0}$, формул $(3.15),(3.18)$ и неравенства $\widetilde{q}(1-p)<1$. Отсюда, учитывая определение (3.13) функции $x_{\gamma}$, получаем утверждение леммы.

Д о к а з а т е л ь с т в о л е м м ы 9. Нахождение функций $x(t) \in$ $\partial D$, минимизирующих квадратическую форму $\left\langle x, A_{\mu}^{-1} x\right\rangle$, проводится на основе равенств (2.3) и (2.4) леммы 1 и замечания 4.

Используя эту лемму и формулы (3.4), (3.22), убеждаемся, что уравнение для минимизирующей центрально-симметричной функции $x(t)$ имеет вид

$$
x(t)=\frac{p}{2} \lambda \int_{0}^{1}\left[(t-s)^{2}-|t-s|+\frac{1}{6}\right]|x(s)|^{p-1} \operatorname{sign} x(s) d s,
$$

где $\lambda>0$, при этом должно быть выполнено условие связи

$$
\int_{0}^{1}|x(t)|^{p} d t=1
$$

Поскольку $x \in \operatorname{Dom}\left(A_{\mu}^{-1}\right)$, то, с учетом (3.7), минимизирующая центрально-симметричная функция дважды дифференцируема всюду на $[0,1]$ и нелинейное интегральное уравнение Хаммерштейна (3.25) сводится к следующей граничной задаче с периодическими граничными условиями:

$$
\left\{\begin{array}{l}
x^{\prime \prime}(t)=-\lambda p|x(t)|^{p-1} \operatorname{sign} x(t), \\
x(0)=x(1), \quad x^{\prime}(0)=x^{\prime}(1), \\
\int_{0}^{1} x(t) d t=0
\end{array}\right.
$$


при этом должно быть выполнено условие нормировки (3.26).

Исследуя граничную задачу (3.27) вместе с условием (3.26) методом работы [32, п. 4.1], убеждаемся, что эта задача имеет в качестве решения однопараметрическое семейство функций. Докажем, что это семейство функций и есть многообразие (3.14).

Покажем, как возникает в качестве экстремальной функиия $x_{0}(t)$, определенная в (3.9)-(3.11).

Решим частный случай задачи (3.27), а именно следуюшую задачу:

$$
\left\{\begin{array}{l}
x^{\prime \prime}(t)=-\lambda p|x(t)|^{p-1} \operatorname{sign} x(t), \\
x(0)=x(1)=0, \quad x^{\prime}(0)=x^{\prime}(1), \\
\int_{0}^{1} x(t) d t=0 .
\end{array}\right.
$$

Задача типа (3.28) была решена в $[32$, п. 4.1], мы применим тот же метод. Записывая первое уравнение из (3.28) в виде $d\left(x^{\prime}(t)\right)^{2}=-2 \lambda d|x(t)|^{p}$, получаем

$$
\left(x^{\prime}(t)\right)^{2}=2 \lambda\left(C-|x(t)|^{p}\right),
$$

где $C \in \mathbf{R}$ - постоянная. Пусть для определенности $x^{\prime}(0)=x^{\prime}(1)>0$, т.е. искомая функиия $x(t)$ возрастает в окрестности нуля. Учитывая замечание 4 , будем искать функцию $x(t)$ с одной переменной знака.

Так как $x(0)=x(1)=0$, то из (3.29) и последнего условия в (3.28) следует, что найдутся точки $0<t_{0}<t_{1}<t_{2}<1$ такие, что $x^{\prime}\left(t_{0}\right)=$ $x^{\prime}\left(t_{2}\right)=0, x\left(t_{1}\right)=0, x\left(t_{0}\right)>0>x\left(t_{2}\right)$, причем точка $t_{0}$ является единственной точкой максимума функции $x(t)$, точка $t_{2}$ - единственной точкой минимума функции $x(t)$, точка $t_{1}$ - единственным нулем этой функции внутри интервала $(0,1)$.

Рассмотрим решение уравнения (3.29) на отрезке $\left[0, t_{1}\right]$, где функция $x(t)$ является неотрицательной. Извлекая квадратный корень, из уравнения (3.29) получаем, что

$$
x^{\prime}(t)= \begin{cases}\sqrt{2 \lambda}\left(C-x^{p}(t)\right)^{1 / 2}, & \text { если } t \in\left[0, t_{0}\right], \\ -\sqrt{2 \lambda}\left(C-x^{p}(t)\right)^{1 / 2}, & \text { если } t \in\left[t_{0}, t_{1}\right] .\end{cases}
$$

Из (3.30) видно, что экстремальная функция $x(t)$ положительна внутри интервала $\left(0, t_{1}\right)$, строго монотонно возрастает на отрезке $\left[0, t_{0}\right]$, строго монотонно убывает на отрезке $\left[t_{0}, t_{1}\right]$, причем

$$
C=x^{p}\left(t_{0}\right)=\frac{\left(x^{\prime}(0)\right)^{2}}{4 \lambda}
$$

Решим граничную задачу на отрезке $\left[0, t_{0}\right]$ :

$$
\left\{\begin{array}{l}
x^{\prime}(t)=\sqrt{2 \lambda} \sqrt{C-x^{p}(t)}, \quad t \in\left[0, t_{0}\right] \\
x(0)=0 .
\end{array}\right.
$$


Из (3.32) получаем для $t \in\left[0, t_{0}\right]$ :

$$
\int_{0}^{x(t)} \frac{d x}{\sqrt{C-x^{p}}}=\sqrt{2 \lambda} t
$$

Отсюда, используя определение неполной бета-функции и ее связь с гипергеометрической функцией Гаусса $F(a, b ; c ; z)$, см. [3, п. 2.5.3] (или формулу 3 из п. 1.2.4 главы 1 книги [28]), приходим к следующему функциональному уравнению для экстремальной функции $x(t)$ на отрезке $\left[0, t_{0}\right]$ :

$$
x(t) F\left(\frac{1}{2}, \frac{1}{p} ; 1+\frac{1}{p} ; \frac{x^{p}(t)}{C}\right)=\sqrt{2 \lambda C} t, \quad t \in\left[0, t_{0}\right] .
$$

Рассмотрим теперь граничную задачу на отрезке $\left[t_{0}, t_{1}\right]$ :

$$
\left\{\begin{array}{l}
x^{\prime}(t)=-\sqrt{2 \lambda} \sqrt{C-x^{p}(t)}, \quad t \in\left[t_{0}, t_{1}\right], \\
x\left(t_{1}\right)=0 .
\end{array}\right.
$$

Из (3.34) получаем для $t \in\left[t_{0}, t_{1}\right]$ :

$$
\int_{0}^{x(t)} \frac{d x}{\sqrt{C-x^{p}}}=\sqrt{2 \lambda}\left(t_{1}-t\right) .
$$

Отсюда, действуя аналогично предыдущему, получаем функциональное уравнение для экстремальной функции $x(t)$ на отрезке $\left[t_{0}, t_{1}\right]$ :

$$
x(t) F\left(\frac{1}{2}, \frac{1}{p} ; 1+\frac{1}{p} ; \frac{x^{p}(t)}{C}\right)=\sqrt{2 \lambda C}\left(t_{1}-t\right), \quad t \in\left[t_{0}, t_{1}\right] .
$$

Положив в уравнениях (3.33) и (3.35) $t=t_{0}$, находим $t_{1}=2 t_{0}$.

Таким образом, из формул (3.33) и (3.35) следует, что решение $\widetilde{x}_{0}(t)$ уравнения $(3.29)$ на отрезке $\left[0, t_{0}\right]$ удовлетворяет уравнению $(3.33)$, а на отрезке $\left[0,2 t_{0}\right]$ симметрично относительно точки $t=t_{0}: \widetilde{x}_{0}(t)=\widetilde{x}_{0}\left(2 t_{0}-t\right)$ для $t \in\left[0,2 t_{0}\right]$.

Совершенно аналогично решается уравнение (3.29) на отрезке $\left[2 t_{0}, 1\right]$, где функция $x(t)$ является неположительной. Здесь также можно доказать, что точка $t_{2}$ находится посередине отрезка $\left[2 t_{0}, 1\right]$, т.е. $t_{2}=$ $t_{0}+1 / 2$. При этом решение $\widetilde{x}_{0}(t)$ уравнения $(3.29)$ на отрезке $\left[t_{2}, 1\right]$ удовлетворяет уравнению

$$
|x(t)| F\left(\frac{1}{2}, \frac{1}{p} ; 1+\frac{1}{p} ; \frac{|x(t)|^{p}}{C}\right)=\sqrt{2 \lambda C}(1-t), \quad t \in\left[t_{2}, 1\right],
$$

с той же самой постоянной $C$, заданной в $(3.31)$, а на отрезке $\left[2 t_{0}, 1\right]$ решение $\widetilde{x}_{0}(t)$ симметрично относительно точки $t=t_{2}$. 
Вывод. Таким образом, из соображений симметрии и непрерывности получаем $t_{0}=1 / 4, t_{1}=1 / 2, t_{2}=3 / 4$. Следовательно, единственным решением задачи (3.28), которое возрастает в окрестности нуля и имеет одну перемену знака, является экстремальная функция $\widetilde{x}_{0}(t)$, удовлетворяющая уравнениям

$$
\begin{cases}x(t) F\left(\frac{1}{2}, \frac{1}{p} ; 1+\frac{1}{p} ; \frac{x^{p}(t)}{C}\right)=\sqrt{2 \lambda C} t, & t \in\left[0, \frac{1}{4}\right], \\ x(t)=x\left(\frac{1}{2}-t\right), & t \in\left[\frac{1}{4}, \frac{1}{2}\right], \\ x_{0}(t)=-x_{0}(1-t), & t \in\left[\frac{1}{2}, 1\right] .\end{cases}
$$

Единственным решением задачи (3.28), которое убывает в окрестности нуля и имеет также одну перемену знака, является, очевидно, функция $-\widetilde{x}_{0}(t), t \in[0,1]$.

Используя условие нормировки (3.26), докажем, что в формуле (3.36) значения постоянных $C$ и $\lambda$ определяются по формуле (3.8), т.е. что $C=C_{0}, \lambda=\lambda_{0}$.

Умножая почленно первое уравнение в $(3.28)$ на $x(t)$ и интегрируя полученное равенство по $t$ от 0 до 1 , находим с учетом (3.26)

$$
\left\langle\widetilde{x}_{0}, A_{\mu}^{-1} \widetilde{x}_{0}\right\rangle=-\int_{0}^{1} \widetilde{x}_{0}(t) \widetilde{x}_{0}^{\prime \prime}(t) d t=\lambda p .
$$

Интегрируя по частям и используя симметрию функции $\widetilde{x}_{0}(t)$ и равенства $\widetilde{x}_{0}(0)=\widetilde{x}_{0}(1)=0$, из формулы $(3.37)$ получаем

$$
\int_{0}^{1 / 4}\left(\widetilde{x}_{0}^{\prime}(t)\right)^{2} d t=\frac{1}{4} \int_{0}^{1}\left(\widetilde{x}_{0}^{\prime}(t)\right)^{2} d t=-\frac{1}{4} \int_{0}^{1} \widetilde{x}_{0}(t) \widetilde{x}_{0}^{\prime \prime}(t) d t=\lambda \frac{p}{4} .
$$

С учетом симметрии функции $\widetilde{x}_{0}(t)$, условие $(3.26)$ принимает вид

$$
\int_{0}^{1 / 4} \widetilde{x}_{0}^{p}(t) d t=\frac{1}{4}
$$

Интегрируя почленно первое уравнение в (3.30) по $t$ от 0 до $t_{0}=1 / 4$ и используя (3.38) и (3.39), получаем равенства

$$
C=4 \int_{0}^{1 / 4} \widetilde{x}_{0}^{p}(t) d t+\frac{2}{\lambda} \int_{0}^{1 / 4}\left(\widetilde{x}_{0}^{\prime}(t)\right)^{2} d t=1+\frac{p}{2} .
$$

Таким образом, равенство $C=C_{0}$ доказано.

Положив в первом уравнении в (3.36) $t=1 / 4$ и учитывая, что $\widetilde{x}_{0}(1 / 4)=C^{1 / p}($ см. $(3.31))$, имеем:

$$
C^{1 / p-1 / 2} F\left(\frac{1}{2}, \frac{1}{p} ; 1+\frac{1}{p} ; 1\right)=\frac{\sqrt{\lambda}}{2 \sqrt{2}} .
$$


Используя формулу 15.1 .20 из справочника [1, гл. 15], находим

$$
F\left(\frac{1}{2}, \frac{1}{p} ; 1+\frac{1}{p} ; 1\right)=\sqrt{\pi} \frac{\Gamma(1+1 / p)}{\Gamma(1 / 2+1 / p)} .
$$

Подставляя (3.40) и (3.42) в (3.41), получаем $\lambda=\lambda_{0}$.

Итак, мы доказали, что экстремальная функция $\widetilde{x}_{0}(t)$ тождественно равна функции $x_{0}(t)$, определенной в $(3.9)-(3.11)$.

Из равенства (3.37) с $\lambda=\lambda_{0}$ и формулы (1.17) следует соотношение $\left\langle x_{0}, A_{\mu}^{-1} x_{0}\right\rangle=\sigma^{-2}(p)$. Таким образом, минимум в экстремальной задаче (3.17) достигается на функции $x_{0}(t)$ и равен $\sigma^{-2}(p)$.

Несложно показать, что, в силу симметрии, для остальных функций $x_{\gamma} \in M_{0}$ выполнены равенства $\left\langle x_{\gamma}, A_{\mu}^{-1} x_{\gamma}\right\rangle=\sigma^{-2}(p), \int_{0}^{1}\left|x_{\gamma}(t)\right|^{p} d t=1$, $0<\gamma<1$.

Также можно доказать, что минимум в экстремальной задаче (3.17) достигается только на функциях из $M_{0}$. В частности, укажем, что функция $x_{\gamma} \in M_{0}, 0<\gamma<1$, является решением граничной задачи (3.27) с $\lambda=\lambda_{0}$ и дополнительным условием $x(0)=x(1)=h_{\gamma} \neq 0$, где $\left|h_{\gamma}\right| \leqslant(1+p / 2)^{1 / p}$. Лемма 9 доказана.

Применение теоремы 1 и леммы 9 дает нам утверждение теоремы 2. Теорема 2 доказана.

Предложение 2. (i) Справедливо равенство для норм экстремальньх функиий, заданных в (3.13):

$$
\left\|x_{\gamma}\right\|_{L^{2}}=\left\|x_{0}\right\|_{L^{2}} \quad \text { для любого } \gamma \in[0,1] .
$$

(ii) Многообразие $M_{0}$, заданное в (3.14) и рассматриваемое в гильбертовом пространстве $L_{0}^{2}$, представляет собой плоскую кривую окружность радиуса $\left\|x_{0}\right\|_{L^{2}}$, чентр которой совпадает с нулем пространства $L_{0}^{2}$. Длина кривой $M_{0}$ выражается формулой

$$
\operatorname{mes}\left(M_{0}\right)=2 \pi\left\|x_{0}\right\|_{L^{2}} \text {. }
$$

(iii) Справедливо равенство для норм производных функций:

$$
\left\|x_{\gamma}^{\prime}\right\|_{L^{2}}=\left\|x_{0}^{\prime}\right\|_{L^{2}} \quad \text { для любого } \gamma \in[0,1] .
$$

Д о к а з а т е л ь с т в о. Согласно формуле (3.13), каждая экстремальная функция $x_{\gamma} \in M_{0}$ определяется посредством сдвига периодической функции $\widehat{x}_{0}(t)$ на число $\gamma \in[0,1]$. Поскольку $\widehat{x}_{0}(t)$ имеет период 1 , то отсюда следует равенство (3.43) для норм в $L_{0}^{2}$. Утверждение (i) предложения 2 доказано.

Докажем второе утверждение. Используя определения (3.10), (3.11), (3.13), несложно убедиться, что для всех $\gamma \in[0,1 / 2]$ выполнено равенство

$$
x_{\gamma}(t)=-x_{\gamma+1 / 2}(t), \quad t \in[0,1] .
$$


Из формул (3.43), (3.46) вытекают равенства

$$
\left\|x_{\gamma}-x_{\gamma+1 / 2}\right\|_{L^{2}}=2\left\|x_{\gamma}\right\|_{L^{2}}=\left\|x_{0}\right\|_{L^{2}} \quad \text { для всех } \gamma \in[0,1 / 2] .
$$

Учитывая формулу (3.47), заключаем, что для любого $\gamma \in[0,1 / 2]$ точки $x_{\gamma}, \mathbf{0}$, и $x_{\gamma+1 / 2}$ лежат на одной прямой, причем $x_{\gamma}$ и $x_{\gamma+1 / 2}$ лежат на одинаковом расстоянии от $\mathbf{0}$ по разные стороны от $\mathbf{0}$. Таким образом, в силу (3.43), (3.47), замкнутая компактная кривая $M_{0}$ представляет собой центрально-симметричное множество, расположенное на сфере радиуса $\left\|x_{0}\right\|_{L^{2}}$ в гильбертовом пространстве $L_{0}^{2}$.

Используя это свойство $M_{0}$ и учитывая тот факт, что многообразие $M_{0}$ построено в результате сдвигов на числа $\gamma \in[0,1]$ одной и той же периодической функции $\widehat{x}_{0}(t)$, можно показать, что $M_{0}$ является плоской кривой, а именно, окружностью радиуса $\left\|x_{0}\right\|_{L^{2}}$, центр которой совпадает с 0. Отсюда непосредственно вытекает формула (3.44). Утверждение (ii) доказано.

Докажем утверждение (iii). Производная непрерывно дифференцируемой периодической функции $\widehat{x}_{0}(t)$ с периодом 1 является периодической непрерывной функцией $\widehat{x}_{0}^{\prime}(t)$ с периодом 1 . Отсюда и из $(3.13)$ следует формула (3.45). Предложение 2 доказано.

\section{4. Случаи $p=1$ и $p=2$. Доказательство следствия 1.}

4.1. Доказательство формулы (1.18). На протяжении данного пункта $p=1$. Таким образом, согласно разделу 3 , основным банаховым пространством, на котором рассматривается гауссовская мера $\mu_{\xi}$, является гильбертово пространство $\left(L_{0}^{2},\|\cdot\|_{L^{2}}\right)$, см. $(3.1)-(3.3)$; в качестве сопряженного пространства берется также пространство $L_{0}^{2},\langle\cdot, \cdot\rangle-$ скалярное произведение в $L_{0}^{2}$, см. (3.21).

Предложение 3. Экстремаль $x_{0}$ состоит из двух параболических кривых и задается формулой

$$
\begin{aligned}
& x_{0}(t)=12 t-24 t^{2}, \quad \text { если } t \in\left[0, \frac{1}{2}\right], \\
& x_{0}(t)=-x_{0}(1-t), \quad \text { если } t \in\left[\frac{1}{2}, 1\right],
\end{aligned}
$$

при этом

$$
\lambda_{0}=\sigma^{-2}(1)=48, \quad C_{0}=\frac{3}{2} .
$$

Д о к а з а т е л ь с т в о. Формулы (4.2) следуют непосредственно из соотношений (1.17) и (3.8). Докажем (4.1). Положив $a=1 / 2$ в формуле 15.1.13 из [1, гл. 15], находим

$$
F\left(\frac{1}{2}, 1 ; 2 ; z\right)=\frac{2}{1+\sqrt{1-z}}, \quad|z| \leqslant 1 .
$$


Используя формулы (4.2), (4.3) и уравнение (3.9), записанное при $p=1$, убеждаемся, что экстремальная функция $x_{0}$ удовлетворяет следующему уравнению:

$$
x(t)=6 t\left[1+\sqrt{1-\frac{2}{3} x(t)}\right], \quad t \in\left[0, \frac{1}{4}\right] .
$$

Решая уравнение (4.4), находим $x_{0}(t)=24 t(1 / 2-t), t \in[0,1 / 4]$. Отсюда, с учетом определения (3.10), (3.11), получаем формулу (4.1). Предложение 3 доказано.

Пусть функции $x_{\gamma}, \gamma \in[0,1]$, и многообразие $M_{0}$, заданные в (3.13), (3.14), построены по функции (4.1). Вычислим значения $c\left(x_{\gamma}\right)$ функционала, определенного в (1.8)-(1.10).

Лемма 11. Для каждого $\gamma \in[0,1]$ выполнено равенство

$$
c\left(x_{\gamma}\right)=\frac{\sqrt{5}}{\sqrt{6}}
$$

Доказательство леммы 11 основано на нескольких вспомогательных утверждениях.

Ради краткости обозначим $n_{\gamma}:=n_{x_{\gamma}}, n_{\gamma}^{*}:=n_{x_{\gamma}}^{*}, B_{\gamma}:=B_{x_{\gamma}}, N_{\gamma}:=$ $N_{x_{\gamma}}, M_{\gamma}:=M_{x_{\gamma}}$ и т.д. Вычисления в гильбертовом пространстве $L_{0}^{2}$ проводить легче. Здесь можно отождествить $n_{\gamma}^{*} \equiv n_{\gamma}$ и положить

$$
N_{\gamma} \equiv N_{\gamma}^{*}=\left\{y \in L_{0}^{2}:\left\langle y, n_{\gamma}\right\rangle=0\right\}
$$

(см. (1.6)). Напомним, что в теореме $1 n_{\gamma} \in L_{0}^{2}-$ это единичный касательный вектор к кривой $M_{0}$ в точке $x_{\gamma}$. Таким образом, теперь, в силу (4.6), вектор $n_{\gamma}$ ортогонален подпространству $N_{\gamma}$.

Лемма 12. Для каждого $\gamma \in[0,1]$ справедливь следуюшие утверждения.

(i) $F^{\prime \prime}\left(x_{\gamma}\right) \equiv 0$ есть нулевой оператор; $B_{\gamma} \equiv \mathscr{I}$ есть единичньй onepamop $u \operatorname{det} B_{\gamma}=1$.

(ii) Выполненьи равенства

$$
\begin{gathered}
\left\langle A n_{\gamma}, n_{\gamma}\right\rangle=\frac{1}{40}, \\
\left\langle A^{N_{\gamma}} A^{-1} x_{\gamma}, A^{-1} x_{\gamma}\right\rangle=\left\langle x_{\gamma}, A^{-1} x_{\gamma}\right\rangle=\sigma^{-2}(1)=48 .
\end{gathered}
$$

Д о к а з а т е л ь с т в о. Зафиксируем $\gamma \in[0,1]$. Известно, что при $p=1$ вторая производная по Фреше функционала (1.14) есть нулевой оператор, поэтому $G^{\prime \prime}\left(x_{\gamma}\right) \equiv 0$ (ср. с (3.24)). Отсюда, в силу (1.5), получаем, что $F^{\prime \prime}\left(x_{\gamma}\right) \equiv 0$ есть нулевой оператор. Используя формулу (1.9), заключаем, что $B_{\gamma} \equiv \mathscr{I}$ есть единичный оператор, детерминант которого равен 1 по определению. Утверждение (i) доказано. 
Формулы (4.7), (4.8) докажем только для $\gamma=0$, случай общего $\gamma$ рассматривается аналогично с учетом предложения 2 .

Согласно определению (см. $[22$, гл. $3, \S 3$, п. 2]), касательный вектор к кривой $(3.14)$ в точке $x_{0}$ имеет вид $\left.\frac{d x_{\gamma}}{d \gamma}\right|_{\gamma=0}$. Используя тот факт, что параметр $\gamma$ в формулах (3.13), (3.14) является параметром сдвига, находим $\left.\frac{d x_{\gamma}}{d \gamma}\right|_{\gamma=0}=x_{0}^{\prime}(t), t \in[0,1]$. Согласно формуле (4.1), получаем

$$
\begin{aligned}
& x_{0}^{\prime}(t)=12-48 t, \quad \text { если } t \in\left[0, \frac{1}{2}\right], \\
& x_{0}^{\prime}(t)=x_{0}^{\prime}(1-t), \quad \text { если } t \in\left[\frac{1}{2}, 1\right] .
\end{aligned}
$$

Как видим, $x_{0}^{\prime}(t) \in L_{0}^{2}$; учитывая (4.9), несложно подсчитать, что $\left\|x_{0}^{\prime}(t)\right\|_{L^{2}}=\sqrt{48}$. Таким образом, единичный касательный вектор $n_{0}$ к кривой $M_{0}$ в точке $x_{0}$ имеет вид

$$
n_{0}(t)=\frac{x_{0}^{\prime}(t)}{\sqrt{48}}, \quad t \in[0,1]
$$

Легко непосредственно проверить, что (как и должно быть в силу (4.6) при $\gamma=0)$ выполнено равенство

$$
\left\langle x_{0}, n_{0}\right\rangle=0 .
$$

Используя соотношения (3.6), (3.7) и (4.10), находим

$$
y_{0}(t):=A n_{0}(t)=-\frac{1}{\sqrt{48}} \int_{0}^{t} x_{0}(s) d s .
$$

Учитывая формулы (4.10), (4.12) и интегрируя по частям, получаем

$$
\begin{aligned}
\left\langle A n_{0}, n_{0}\right\rangle & =\frac{1}{\sqrt{48}} \int_{0}^{1} y_{0}(t) x_{0}^{\prime}(t) d t=-\frac{1}{\sqrt{48}} \int_{0}^{1} x_{0}(t) y_{0}^{\prime}(t) d t \\
& =\frac{1}{48} \int_{0}^{1} x_{0}^{2}(t) d t=\frac{1}{40},
\end{aligned}
$$

так как, в силу (4.1), $\int_{0}^{1} x_{0}^{2}(t) d t=6 / 5$. Равенство (4.7) доказано для $\gamma=0$.

Согласно формулам (2.17) и (4.11), находим

$$
A^{N_{0}} A^{-1} x_{0}=x_{0}-\frac{\left\langle n_{0}, x_{0}\right\rangle}{\left\langle A n_{0}, n_{0}\right\rangle} A n_{0}=x_{0} .
$$

Отсюда, с учетом леммы 9, получаем равенства (4.8) при $\gamma=0$. Лемма 12 доказана. 
Док а з а те ль с тв о ле м мы 11. Из леммы 12 и соотношений (1.8)-(1.10) находим

$$
c\left(x_{\gamma}\right)=\left[\left\langle A n_{\gamma}, n_{\gamma}\right\rangle\left\langle x_{\gamma}, A^{-1} x_{\gamma}\right\rangle\right]^{-1 / 2}=\frac{\sqrt{5}}{\sqrt{6}} .
$$

Формула (4.5) доказана.

Д о к а з а т е л ь с т в о фо о м у лы (1.18). Используя теоремы 1,2 и лемму 11 , получаем соотношение (1.18), поскольку в силу предложения 2 и формулы $\left\|x_{0}\right\|_{L^{2}}=\sqrt{6} / \sqrt{5}$ выполнено равенство mes $\left(M_{0}\right)=$ $2 \pi \sqrt{5} / \sqrt{6}$.

4.2. Доказательство формулы (1.19). На протяжении данного пункта $p=2$. Здесь также гауссовская мера $\mu_{\xi}$ рассматривается на гильбертовом пространстве $L_{0}^{2}$.

Предложение 4. Экстремаль $x_{0}$ задается формулой

$$
x_{0}(t)=\sqrt{2} \sin (2 \pi t), \quad t \in[0,1],
$$

при этом

$$
\lambda_{0}=2 \pi^{2}, \quad \sigma^{-2}(2)=4 \pi^{2}, \quad C_{0}=2 .
$$

Д о к а з а т е л ь с т в о. Формулы (4.14) следуют непосредственно из соотношений (1.17) и (3.8). Докажем (4.13). Согласно формуле 15.1.6 из $[1$, гл. 15$]$, находим

$$
F\left(\frac{1}{2}, \frac{1}{2} ; \frac{3}{2} ; z\right)=\frac{\arcsin \sqrt{z}}{\sqrt{z}}, \quad|z| \leqslant 1 .
$$

Используя формулы (4.14), (4.15) и уравнение (3.9), записанное при $p=2$, убеждаемся, что экстремальная функция $x_{0}$ удовлетворяет следующему уравнению:

$$
\arcsin \left(\frac{x(t)}{\sqrt{2}}\right)=2 \pi t, \quad t \in\left[0, \frac{1}{4}\right] .
$$

Из (4.16) находим $x_{0}(t)=\sqrt{2} \sin (2 \pi t), t \in[0,1 / 4]$. Отсюда, с учетом определения (3.10), (3.11), получаем формулу (4.14). Предложение 4 доказано.

Дальнейшие вычисления можно провести так же, как в случае $p=$ 1, но ради краткости изложения мы используем следующий результат (см. [13] или теорему 3.8 при $r=2$ в [26]).

Теорема 3. Пусть $X=\left(X_{1}, X_{2}, \ldots\right)-$ гауссовский элемент в координатном гильбертовом пространстве $\left(\ell^{2},\|\cdot\|_{2}\right)$ со средним нуль и невырожденньлм ковариационньлм оператором $A: \ell^{2} \rightarrow \ell^{2}$. Пусть ядерный оператор А имеет собственные значения

$$
\sigma_{1}^{2}=\sigma_{2}^{2}>\sigma_{3}^{2} \geqslant \sigma_{4}^{2} \geqslant \cdots>0 .
$$


Тогда минимум выражения $\left\langle x, A^{-1} x\right\rangle$ на множестве $\left\{x \in \ell^{2}:\|x\|_{2} \geqslant 1\right\}$ равен $\sigma_{1}^{-2}$ и достигается на одномерном многообразии $\left\{y \in \ell^{2}: y_{1}^{2}+y_{2}^{2}=\right.$ $\left.1, y_{3}=y_{4}=\cdots=0\right\}$. Справедливо соотношение

$$
\mathbf{P}\left\{\|X\|_{2}>u\right\}=\exp \left\{-\frac{u^{2}}{2 \sigma_{1}^{2}}\right\} \prod_{j=3}^{\infty}\left(1-\frac{\sigma_{j}^{2}}{\sigma_{1}^{2}}\right)^{-1 / 2}(1+o(1)), \quad u \rightarrow \infty .
$$

Учитывая формулы (3.6), (3.7) и результат пункта (г) из [14, ч. 3 , гл. 2, № 2.9], убеждаемся, что все собственные значения ковариационного оператора $A_{\mu}: L_{0}^{2} \rightarrow L_{0}^{2}$ двукратны и имеют вид

$$
\sigma_{2 n+1}^{2}=\sigma_{2 n+2}^{2}=\frac{1}{4 \pi^{2}(n+1)^{2}}, \quad n=0,1,2, \ldots
$$

Используя (4.18) и формулу 7 из $[28$, гл. 6, п. 6.2 .1$]$, находим

$$
\prod_{j=3}^{\infty}\left(1-\frac{\sigma_{j}^{2}}{\sigma_{1}^{2}}\right)=\left[\prod_{k=2}^{\infty}\left(1-\frac{1}{k^{2}}\right)\right]^{2}=\frac{1}{4} .
$$

Подставляя (4.19) в (4.17) и учитывая, что $\sigma_{1}^{-2}=4 \pi^{2}$, получаем формулу (1.19). Следствие 1 доказано.

\section{СПИСОК ЛИТЕРАТУРЫ}

1. Абрамовии М., Стиган И. (ред.). Справочник по специальным функциям. М.: Наука, 1979, 830 c.

2. Алексеев B. М., Тихомиров В. М., Фомин С. В. Оптимальное управление. М.: Наука, 1978, 429 с.

3. Бейтмен Г., Эрдейи А. Высшие трансцендентные функции: Гипергеометрическая функция. Функции Лежандра. М.: Наука, 1973, 294 с.

4. Боровков A.A., Могульский A. А. О вероятностях больших уклонений в топологических пространствах, I, II. Сибир. матем. журн., 1978, т. 19, № 5, с. 988-1004; 1980, т. 21, № 5, с. 12-26.

5. Вайнберг M. М. Вариационный метод и метод монотонных операторов в теории нелинейных операторов. М.: Наука, 1972, 416 с.

6. Вахания Н. Н., Тариеладзе В.И., Чобанян С. А. Вероятностные распределения в банаховых пространствах. М.: Наука, 1985, 369 с.

7. Вентчель А. Д., Фрейдлин М. И. Флуктуации в динамических системах под действием малых случайных возмущений. М.: Наука, 1979, 424 с.

8. Вентиель А. Д. Предельные теоремы о больших уклонениях для марковских случайных процессов. М.: Наука, 1986, 175 с.

9. Гихман И. И., Скороход А.В. Теория случайных процессов, т. 1. М., Наука, 1971, $664 \mathrm{c}$.

10. Го Х.-С. Гауссовские меры в банаховых пространствах. М.: Мир, 1979, 176 с.

11. Гохберг И. Ц., Крейн М. Г. Введение в теорию линейных несамосопряженных операторов в гильбертовом пространстве. М.: Наука, 1965, 448 с.

12. Данфорд Н., Швари Дж. Т. Линейные операторы. Общая теория. М.: УРСС, 2004, $895 \mathrm{c.}$ 
13. Золотарев В. М. Об одной вероятностной задаче. - Теория вероятн. и ее примен., 1961, т. 6, в. 2, с. 219-222.

14. Камке Э. Справочник по обыкновенным дифференциальным уравнениям, М.: Наука, 1965, 703 с.

15. Канторович Л. В., Акилов Г. П. Функциональный анализ. М.: Наука, 1977, 741 с.

16. Коддингтон Э. А., Левинсон $H$. Теория обыкновенных дифференциальных уравнений. М.: УРСС, 2007, 474 с.

17. Красносельский М. А., Забрейко П. П., Пустьльник Е. И., Соболевский П. Е. Интегральные операторы в пространствах суммируемых функций. М.: Наука, 1966, $499 \mathrm{c.}$

18. Крейн С. Г. (ред.). Функциональный анализ. М.: Наука, 1972, 544 с.

19. Ланкастер П. Теория матриц. М.: Наука, 1982, 269 с.

20. Лифшич М. А. Гауссовские случайные функции. Киев: ТВиМС, 1995, 246 с.

21. Мартынов Г. В. Критерии омега-квадрат. М.: Наука, 1978, 79 с.

22. Мищенко A. C., Фоменко A. T. Курс дифференциальной геометрии и топологии. М.: Изд-во Моск. ун-та, 1980, 439 с.

23. Назаров А. И. О точной константе в обобщенном неравенстве Пуанкаре. - Проблемы математического анализа, 2002, в. 24, с. 155-179.

24. Никитин Я. Ю. Асимптотическая эффективность непараметрических критериев. М.: Наука, 1995, 238 c.

25. Питербарг В. И. Асимптотические методы в теории гауссовских случайных процессов и полей. М.: Изд-во Моск. ун-та, 1988, 175 с.

26. Питербарг В. И., Фаталов В. Р. Метод Лапласа для вероятностных мер в банаховых пространствах. - Успехи матем. наук, 1995, т. 50, № 6, с. 57-150.

27. Пич А. Операторные идеалы. М.: Мир, 1982, 536 с.

28. Прудников А. П., Брычков Ю. А., Маричев О. И. Интегралы и ряды: Элементарные функции. М.: Наука, 1981, 798 с.

29. Фаталов В.Р. Точные асимптотики больших уклонений для гауссовских мер в гильбертовом пространстве. - Изв. НАН Армении, 1992, т. 27, № 5, с. 43-57.

30. Фаталов В.Р. Асимптотики вероятностей больших уклонений гауссовских полей. - Изв. НАН Армении, 1992, т. 27, № 6, с. 59-81; 1993, т. 28, № 5, с. 32-55.

31. Фаталов В.Р. Большие уклонения для гауссовских процессов в гёльдеровской норме. - Изв. РАН, 2003, т. 67, № 5, с. 207-224.

32. Фаталов B.P. Точные асимптотики типа Лапласа для умеренных уклонений распределений сумм независимых банаховозначных случайных элементов, Теория вероятн. и ее примен., 2003, т. 48, № 4, с. 720-744; исправления: 2006, т. 51, № 3 , с. $634-636$.

33. Фаталов В. Р. Метод Лапласа для малых уклонений гауссовских процессов типа винеровского. - Матем. сб., 2005, т. 196, № 4, с. 135-160.

34. Фаталов В.Р. Большие уклонения гауссовских мер в пространствах $l^{p}$ и $L^{p}$, $p \geqslant 2$. - Теория вероятн. и ее примен., 1996 , т. 41 , в. 3 , с. $682-689$; исправления: 2006, т. 51, в. 3 , с. $634-636$.

35. Фаталов B.P. Асимптотики больших уклонений винеровских полей в $L^{p}$-норме, нелинейные уравнения Хаммерштейна и гиперболические краевые задачи высокого порядка. - Теория вероятн. и ее примен., 2002, т. 47, в. 4, с. 710-726; исправления: 2006, т. 51, в. 3, с. 634-636.

36. Фаталов В.Р. Точные асимптотики типа Лапласа для гауссовской меры Боголюбова. - Теорет. и матем. физ., 2011, т. 168, № 2, с. 299-340.

37. Федорюк М. В. Асимптотика: интегралы и ряды. М.: Наука, 1987, 544 с.

38. Фейнман P., Хибс А. Квантовая механика и интегралы по траекториям, М.: Мир, $1968,382 \mathrm{c}$.

39. Azencott $R$. Formule de Taylor stochastique et développement asymptotique d'intégrales de Feynman. - Lecture Notes in Math., 1982, v. 921, p. 237-285.

40. Ben Arous $G$. Méthodes de Laplace et de la phase stationnaire sur l'espace de Wiener. - Stochastics, 1988, v. 25, № 3, p. 125-153.

41. Bolthausen E. Laplace approximations for sums of independent random vectors. I, II. - Probab. Theory Related Fields, 1986, v. 72, № 2, p. 305-318; 1987, v. 76, № 2, p. $167-206$. 
42. Bolthausen E., Deuschel J.-D., Tamura Y. Laplace approximations for large deviations of nonreversible Markov processes. The nondegenerate case. - Ann. Probab., 1995 , v. 23, № 1, p. 236-267.

43. Chevet $S$. Gaussian measures and large deviations. - Lecture Notes in Math., 1983, v. 990 , p. $30-46$.

44. Darling D. A. On the supremum of a certain Gaussian process. - Ann. Probab., 1983, v. 11, № 3, p. 803-806.

45. Davies I. M. Laplace asymptotic expansions for Gaussian functional integrals. Electron. J. Probab., 1998, v. 3, № 13, p. 1-19.

46. Donsker M.D., Varadhan S.R.S. Asymptotic evaluation of certain Markov process expectations for large time. I, II, III, IV. - Comm. Pure Appl. Math., 1975, v. 28, p. $1-47 ; 1975$, v. 28 , p. $279-301 ; 1976$, v. 29 , p. $389-461 ; 1983$, v. 36 , p. $525-565$.

47. Ellis R. S., Rosen J.S. Asymptotic analysis of Gaussian integrals. I: Isolated minimum points. II: Manifold of minimum points. - Trans. Amer. Math. Soc., 1982, v. 273, № 2, p. 447-481; Comm. Math. Phys., 1981, v. 82, № 2, p. 153-181.

48. Fatalov V.R. The Laplace method for computing exact asymptotics of distributions of integral statistics. - Math. Methods Statist., 1999, v. 8, № 4, p. 510-535.

49. Henze N., Nikitin Ya. Yu. Watson-type goodness-of-fit tests based onthe integrated empirical process. - Math. Methods Statist., 2002, v. 11, № 2, p. 183-202.

50. Kuelbs $J$. Large deviation probabilities and dominating points for open convex sets: nonlogarithmic behavior. - Ann. Probab., 2000, v. 28, № 3, p. 1259-1279.

51. Kusuoka S., Tamura Y. Precise estimate for large deviation of Donsker-Varadhan type. - J. Fac. Sci. Univ. Tokyo, Sect. IA Math., 1991, v. 38, № 3, p. 533-565.

52. Kusuoka S., Liang $S$. Laplace approximations for sums of independent random vectors. - Probab. Theory Related Fields, 2000, v. 116, № 2, p. 221-238.

53. Liang S. Laplace approximations for sums of independent random vectors: The degenerate case. - J. Math. Sci. Univ. Tokyo, 2000, v. 7, № 2, p. 195-220.

54. Lifshits M. A. Gaussian large deviations of a smooth seminorm. - Proceedings of the Sixth USSR-Japan Symposium on Probability Theory and Mathematical Statistics, River Edge, World Scientific, 1992, p. 193-201.

55. Lifshits M.A., Nazarov A.I., Nikitin Ya. Yu. Tail behavior of anisotropic norms for Gaussian random fields. - C. R. Math. Acad. Sci. Paris, 2003, v. 336, № 1, p. 85-88.

56. Pietsch A. Eigenvalues and $s$-Numbers. Cambridge: Cambridge Univ. Press, 1987, 360 p.

57. Podkorytova O.A. On tail asymptotics for $L^{1}$-norm of centered Brownian bridge. Matematiche (Catania), 1998, v. 53, № 1, p. 3-9.

58. Rovira C., Tindel S. Sharp Laplace asymptotics for a parabolic SPDE. - Stochastics Stochastics Rep., 2000, v. 69, № 1-2, 11-30.

59. Rovira C., Tindel S. Sharp Laplace asymptotics for a hyperbolic SPDE. - Stochastic Analysis and Related Topics, VII (Kusadasi, 1998). Boston: Birkhäuser, 2001, p. 225244. (Progr. Probab., v. 48.)

60. Simon B. Functional Integration and Quantum Physics. New York: Academic Press, 1979, $296 \mathrm{p}$.

61. Talagrand M. Small tails for the supremum of a Gaussian processes. - Ann. Inst. H. Poincaré, 1988, v. 24, № 2, p. 307-315.

62. Watson G. S. Goodness-of-fit tests on a circle. — Biometrika, 1961, v. 48, № 1, p. 109114. 